ESAIM: M2AN 50 (2016) 1167-1192

DOI: $10.1051 / \mathrm{m} 2 \mathrm{an} / 2015074$
ESAIM: Mathematical Modelling and Numerical Analysis

www.esaim-m2an.org

\title{
A TWO-FLUID FOUR-EQUATION MODEL WITH INSTANTANEOUS THERMODYNAMICAL EQUILIBRIUM
}

\author{
Alexandre Morin ${ }^{1,2}$ And TORE FlåTten ${ }^{2}$
}

\begin{abstract}
We consider an equilibrium version of a common two-fluid model for pipe flow, containing one mixture mass equation and one mixture energy equation. This model can be derived from a fiveequation model with instantaneous thermal equilibrium, to which additional phase relaxation terms are added. An original contribution of this paper is a quasilinear formulation of the instantaneous phase relaxation limit. From this, the mixture sound speed intrinsic to the model can be extracted. This allows us to directly prove some subcharacteristic conditions with respect to a previously established model hierarchy of different relaxation processes. These subcharacteristic conditions reveal the fundamental insight of this paper; in the hierarchy, thermodynamic versus velocity relaxation both reduce the mixture sound velocity with a factor that is independent of whether the other type of relaxation has been performed.
\end{abstract}

Mathematics Subject Classification. 76T10, 35L65.

Received March 28, 2015. Revised August 7, 2015. Accepted September 18, 2015.

\section{INTRODUCTION}

One-dimensional two-phase flows in pipelines may be modelled using the two-fluid approach $[32,35,44,46]$. The two-fluid model is characterised by the fact that it has two separate momentum equations. Therefore, the phase velocities are independent from each other, as opposed to drift-flux models $[1,14,17,33,39]$ where there is only one momentum equation for the mixture. A six-equation version of the two-fluid model is used for example in the nuclear industry $[6,49]$. In this version, the phases are in mechanical equilibrium - they are at the same pressure at all time - but not in chemical and thermal equilibrium. A five-equation version has been chosen for pipeline flow simulation [4], in which the phases are assumed to be in mechanical and thermal equilibrium. A seven-equation version, where the phases are allowed to be totally out of equilibrium - both have their own pressure, temperature and chemical potential - has also been derived [3,38]. One quality of the latter model is that it avoids the loss of hyperbolicity $[19,45]$ commonly associated with the six-equation model.

\footnotetext{
Keywords and phrases. Two-phase flows, relaxation, two-fluid model, subcharacteristic condition.

1 Department of Energy and Process Engineering, Norwegian University of Science and Technology (NTNU), Kolbjørn Hejes vei 1B, NO-7491 Trondheim, Norway.

2 SINTEF Materials and Chemistry, P. O. Box 4760 Sluppen, NO-7465 Trondheim, Norway. alexandre.morin@sintef.no; Tore.Flatten@sintef .no
} 
Relaxation source terms may be added to the models to bring them towards some equilibrium at a finite rate. This has been studied for example by $[15,23,29,34,36-38,48]$. In this setting, the various equilibrium models may be obtained from each other by taking the limit of infinitely stiff source terms.

For the simulation of the two-phase flow of a mixture with phase change (see for example [14,20,21]), the equation of state plays an important role. In this context, a general framework for constructing state equations for phase transitions was presented by Helluy and Seguin [22].

For two-phase mixtures of $\mathrm{CO}_{2}$, the Span-Wagner equation of state [43] is highly accurate. However it is an equilibrium equation of state, which means that the fluid-dynamical model must handle a mixture that is at equilibrium at all time. Munkejord and Hammer [31] used the two-fluid five-equation model presented in [29], where the relaxation to chemical equilibrium is performed between each time step through a fractional step approach. In the present paper, we derive the four-equation model from the five-equation model, where we replace the individual phase mass-equations by a mixture mass equation and an instantaneous chemical equilibrium assumption. Such a two-fluid model was considered in [40], and this model forms the basis for our current paper. Herein, the momentum-exchange terms require a careful treatment when the phase change becomes instantaneous. This allows us to find a convenient quasilinear form of the model. The formulation of the model is independent of the equation of state. An example of numerical simulations with the Span-Wagner equation of state can be found in [20].

The inclusion of stiff relaxation terms in a model will modify its wave structure. The linear stability condition of the equilibrium limit is termed the subcharacteristic condition $[9,15,26,29,34,42]$. A consequence of this condition is that the velocities of the waves of an equilibrium system cannot exceed the maximum velocities of the relaxation system. Several recent works have systematically investigated various two-phase flow models with the aim of verifying this condition $[15,17,27,29]$. In particular, Ferrer et al. [29] concentrated on velocity and thermal relaxation. A main observation of that paper is that these relaxation processes "commute" in the sense that the sound speed is reduced by the same factor regardless of the order in which the relaxation processes are performed.

The objective of this paper is to extend this analysis to the less tractable phase relaxation process. In particular, we will achieve the following 3 main goals:

(1) Derive a standard quasilinear formulation of the full equilibrium two-fluid model and calculate the mixture sound velocity.

(2) Place our model in the established hierarchy of relaxation models and verify the subcharacteristic conditions.

(3) Extend the commutation principle observed in [29] to include the process of phase relaxation.

It should be noted that in this kind of linear analysis we consistently assume smooth solutions.

For full equilibrium models, where the phasic velocities are a priori set to be equal, strict hyperbolicity holds subject to classical thermodynamical stability assumptions $[2,14]$. However, a well-known and controversial feature of the kind of two-fluid models we consider is their tendency to lose hyperbolicity when the relative velocity between the phases exceed a critical value $[6,10,12,13,35,44,46]$. In fact, unless the velocities in each phase are identical, there seems to be a fundamental incompatibility between the second law of thermodynamics and the hope of obtaining a hyperbolic first-order model [16]. In this paper, we will not put a strong focus on this issue; we simply observe that these models are extensively used in practice although an ultimate formulation has not yet been agreed upon.

In this respect, we also wish to remark that the major analyses of this paper are based on the fluid-dynamical equilibrium state where the phasic velocities are equal. In this case many of the various regularizing terms proposed in the literature disappear, so our main results should still have a rather general validity despite the controversies surrounding the precise model formulation.

The structure of the paper is as follows. In Section 2, we present the five-equation model investigated in [29], to which we add relaxation source terms for phase and momentum transfer. These involve an interfacial momentum velocity, for which we derive a precise expression with the help of entropy considerations. In Section 3, the stiff limit of this model is formulated. In particular, we express the phase change relaxation source terms by means 
TABLE 1. Main symbols.

\begin{tabular}{ll}
\hline Symbol & Signification \\
\hline$c$ & Speed of sound \\
$C_{p}$ & Specific heat capacity at constant pressure \\
$e$ & Internal energy \\
$s$ & Specific entropy \\
$E$ & Phasic total energy $\left(E=\alpha \rho\left(e+1 / 2 v^{2}\right)\right)$ \\
$f_{i}$ & Components of the vector $\boldsymbol{F}$ \\
$\mathcal{F}$ & Velocity relaxation coefficient \\
$\mathcal{K}$ & Chemical relaxation coefficient \\
$p$ & Pressure \\
$T$ & Temperature \\
$u_{i}$ & Components of the vector $\boldsymbol{U}$ \\
$v$ & Velocity \\
$w_{i}$ & Components of the vector $\boldsymbol{W}$ \\
\hline$\alpha$ & Volume fraction \\
$\Gamma$ & First Grüneisen coefficient $\left(\Gamma=1 / \rho(\partial p / \partial e)_{\rho}\right)$ \\
$\varepsilon$ & Perturbation parameter \\
$\mu$ & Chemical potential \\
$\rho$ & Density \\
\hline $\boldsymbol{A}$ & Jacobian \\
$\boldsymbol{B}$ & Coefficient matrix in the non-conservative terms \\
$\boldsymbol{F}$ & Vector of the fluxes \\
$\boldsymbol{U}$ & Vector of the conserved variables \\
$\boldsymbol{W}$ & Vector of the non-conservative variables \\
\hline $\mathrm{g}$ & Gas phase (Subscript) \\
$\ell$ & Liquid phase (Subscript) \\
\hline &
\end{tabular}

of spatial derivatives, so that no algebraic terms remain in the system. Then, in Section 4, the system is written in quasilinear form, which involves finding suitable variables in which to express the rather involved Jacobian of the fluxes. In Section 5, the speed of sound of the model is evaluated, and the expected subcharacteristic conditions with respect to other two-phase flow models are verified. A main result of the present paper is the equation (5.27), which extends the commutation result established in [29] to include the phase relaxation process.

In Section 6, we investigate to which degree an interfacial pressure correction term previously applied to related models $[6,10,12,13,35,46]$ can regularize our current model. Through a perturbation method, similar to the approach followed in $[46,47]$, we conclude that an expression widely used for the full six-equation two-fluid model $[8,13,32,35,45]$ has the precise same regularizing effect also on the phase equilibrium model considered in this paper.

Finally, in Section 7, the results of our paper are summarized. The main symbols used are listed in Table 1. Remaining symbols will be introduced in the text.

\section{The five EQUATIOn MOdel With PHASE RELAXATION}

The two-fluid five-equation model studied by [29] describes a one-dimensional two-phase flow where the pressure and the temperature are kept equal in both phases at all times. This follows from the assumption of instantaneous mechanical and thermal equilibrium. However, the two phases will in general not be in chemical equilibrium. Algebraic relaxation terms representing phase change should then act to attract the phases towards equilibrium. In addition, the drag force between the phases will tend to equalize the phase velocities. After 
addition of phase and velocity relaxations, the five-equation model described in [29] becomes

$$
\begin{gathered}
\frac{\partial \alpha_{\mathrm{g}} \rho_{\mathrm{g}}}{\partial t}+\frac{\partial \alpha_{\mathrm{g}} \rho_{\mathrm{g}} v_{\mathrm{g}}}{\partial x}=\mathcal{K}\left(\mu_{\ell}-\mu_{\mathrm{g}}\right), \\
\frac{\partial \alpha_{\ell} \rho_{\ell}}{\partial t}+\frac{\partial \alpha_{\ell} \rho_{\ell} v_{\ell}}{\partial x}=\mathcal{K}\left(\mu_{\mathrm{g}}-\mu_{\ell}\right), \\
\frac{\partial \alpha_{\mathrm{g}} \rho_{\mathrm{g}} v_{\mathrm{g}}}{\partial t}+\frac{\partial \alpha_{\mathrm{g}} \rho_{\mathrm{g}} v_{\mathrm{g}}^{2}}{\partial x}+\alpha_{\mathrm{g}} \frac{\partial p}{\partial x}=v_{\mathrm{i}} \mathcal{K}\left(\mu_{\ell}-\mu_{\mathrm{g}}\right)+\mathcal{F}\left(v_{\ell}-v_{\mathrm{g}}\right), \\
\frac{\partial \alpha_{\ell} \rho_{\ell} v_{\ell}}{\partial t}+\frac{\partial \alpha_{\ell} \rho_{\ell} v_{\ell}^{2}}{\partial x}+\alpha_{\ell} \frac{\partial p}{\partial x}=v_{\mathrm{i}} \mathcal{K}\left(\mu_{\mathrm{g}}-\mu_{\ell}\right)+\mathcal{F}\left(v_{\mathrm{g}}-v_{\ell}\right), \\
\frac{\partial\left(E_{\mathrm{g}}+E_{\ell}\right)}{\partial t}+\frac{\partial}{\partial x}\left(\left(E_{\mathrm{g}}+\alpha_{\mathrm{g}} p\right) v_{\mathrm{g}}+\left(E_{\ell}+\alpha_{\ell} p\right) v_{\ell}\right)=0,
\end{gathered}
$$

where the total energy $E_{k}$ of phase $k \in\{\mathrm{g}, \ell\}$ is the sum of kinetic and internal energy:

$$
E_{k}=\alpha_{k} \rho_{k}\left(e_{k}+\frac{1}{2} v_{k}^{2}\right)
$$

$\mathcal{F}$ and $\mathcal{K}$ are positive relaxation constants, $\mu$ is the chemical potential, and $v_{\mathrm{i}}$ is some interface velocity. Assuming that the phases are composed of only one component, we may express the chemical potential $\mu_{k}$ of phase $k \in\{\mathrm{g}, \ell\}$ as

$$
\mu_{k}=e_{k}+\frac{p}{\rho_{k}}-T s_{k}
$$

We will first only consider the chemical relaxation and thus set the velocity relaxation coefficient to $\mathcal{F}=0$. We will then have use for $\mathcal{F} \neq 0$ from Section 5 , when we will discuss the drift-flux models.

\subsection{Interfacial momentum velocity}

Following in the footsteps of $[11,18]$, we are able to give an expression for the interface velocity $v_{\mathrm{i}}$ through entropy considerations.

Proposition 2.1. If we assume that the interface velocity $v_{\mathrm{i}}$ is independent of $\mu_{\mathrm{g}}-\mu_{\ell}$, the second law of thermodynamics uniquely determines

$$
v_{\mathrm{i}}=\frac{1}{2}\left(v_{\mathrm{g}}+v_{\ell}\right)
$$

Proof. We will derive the mixture entropy evolution equation, and impose that the source term should be nonnegative. We first derive the kinetic energy evolution equations, by multiplying the momentum equations $(2.3)$ and (2.4) by $v_{\mathrm{g}}$ and $v_{\ell}$, respectively. For the gas phase, after expansion of the derivatives, we obtain

$$
v_{\mathrm{g}}^{2} \frac{\partial \alpha_{\mathrm{g}} \rho_{\mathrm{g}}}{\partial t}+\alpha_{\mathrm{g}} \rho_{\mathrm{g}} v_{\mathrm{g}} \frac{\partial v_{\mathrm{g}}}{\partial t}+v_{\mathrm{g}}^{2} \frac{\partial \alpha_{\mathrm{g}} \rho_{\mathrm{g}} v_{\mathrm{g}}}{\partial x}+\alpha_{\mathrm{g}} \rho_{\mathrm{g}} v_{\mathrm{g}}^{2} \frac{\partial v_{\mathrm{g}}}{\partial x}+\alpha_{\mathrm{g}} v_{\mathrm{g}} \frac{\partial p}{\partial x}=v_{\mathrm{g}} v_{\mathrm{i}} \mathcal{K}\left(\mu_{\ell}-\mu_{\mathrm{g}}\right)
$$

The same applies to the liquid phase. After the use of the mass equations (2.1)-(2.2) and reorganisation, the equations read

$$
\begin{aligned}
& \frac{\partial}{\partial t}\left(\frac{1}{2} \alpha_{\mathrm{g}} \rho_{\mathrm{g}} v_{\mathrm{g}}^{2}\right)+\frac{\partial}{\partial x}\left(\frac{1}{2} \alpha_{\mathrm{g}} \rho_{\mathrm{g}} v_{\mathrm{g}}^{3}\right)+\alpha_{\mathrm{g}} v_{\mathrm{g}} \frac{\partial p}{\partial x}=v_{\mathrm{g}}\left(v_{\mathrm{i}}-\frac{1}{2} v_{\mathrm{g}}\right) \mathcal{K}\left(\mu_{\ell}-\mu_{\mathrm{g}}\right) \\
& \frac{\partial}{\partial t}\left(\frac{1}{2} \alpha_{\ell} \rho_{\ell} v_{\ell}^{2}\right)+\frac{\partial}{\partial x}\left(\frac{1}{2} \alpha_{\ell} \rho_{\ell} v_{\ell}^{3}\right)+\alpha_{\ell} v_{\ell} \frac{\partial p}{\partial x}=v_{\ell}\left(v_{\mathrm{i}}-\frac{1}{2} v_{\ell}\right) \mathcal{K}\left(\mu_{\mathrm{g}}-\mu_{\ell}\right)
\end{aligned}
$$


Using the latter equations, we can now cancel the kinetic energy contribution in the mixture total energy equation (2.5), which gives

$$
\begin{aligned}
\frac{\partial}{\partial t}\left(\alpha_{\mathrm{g}} \rho_{\mathrm{g}} e_{\mathrm{g}}+\alpha_{\ell} \rho_{\ell} e_{\ell}\right)+\frac{\partial}{\partial x}\left(\alpha_{\mathrm{g}} \rho_{\mathrm{g}} e_{\mathrm{g}} v_{\mathrm{g}}\right. & \left.+\alpha_{\ell} \rho_{\ell} e_{\ell} v_{\ell}\right) \\
& +p \frac{\partial \alpha_{\mathrm{g}} v_{\mathrm{g}}}{\partial x}+p \frac{\partial \alpha_{\ell} v_{\ell}}{\partial x}=\left(v_{\mathrm{g}}-v_{\ell}\right)\left(v_{\mathrm{i}}-\frac{1}{2}\left(v_{\mathrm{g}}+v_{\ell}\right)\right) \mathcal{K}\left(\mu_{\mathrm{g}}-\mu_{\ell}\right) .
\end{aligned}
$$

By the mass equations (2.1) and (2.2), we obtain an evolution equation for the material derivatives of the phasic internal energy

$$
\alpha_{\mathrm{g}} \rho_{\mathrm{g}} \frac{D_{\mathrm{g}} e_{\mathrm{g}}}{D t}+\alpha_{\ell} \rho_{\ell} \frac{D_{\ell} e_{\ell}}{D t}+p \frac{\partial \alpha_{\mathrm{g}} v_{\mathrm{g}}}{\partial x}+p \frac{\partial \alpha_{\ell} v_{\ell}}{\partial x}=\left(\left(v_{\mathrm{g}}-v_{\ell}\right)\left(v_{\mathrm{i}}-\frac{1}{2}\left(v_{\mathrm{g}}+v_{\ell}\right)\right)+e_{\mathrm{g}}-e_{\ell}\right) \mathcal{K}\left(\mu_{\mathrm{g}}-\mu_{\ell}\right)
$$

where we have introduced the phase specific material derivative $\frac{D_{k}}{D t}=\frac{\partial}{\partial t}+v_{k} \frac{\partial}{\partial x}$.

Using the fundamental thermodynamic relation

$$
\mathrm{d} e_{k}=\frac{p}{\rho_{k}^{2}} \mathrm{~d} \rho_{k}+T \mathrm{~d} s_{k},
$$

we can transform the previous equation into an entropy equation. First, (2.14) is expressed in terms of material derivatives and substituted in the internal energy equation (2.13)

$$
\begin{aligned}
\alpha_{\mathrm{g}} \rho_{\mathrm{g}}\left(T \frac{D_{\mathrm{g}} s_{\mathrm{g}}}{D t}+\frac{p}{\rho_{\mathrm{g}}^{2}} \frac{D_{\mathrm{g}} \rho_{\mathrm{g}}}{D t}\right)+\alpha_{\ell} \rho_{\ell}\left(T \frac{D_{\ell} s_{\ell}}{D t}+\frac{p}{\rho_{\ell}^{2}} \frac{D_{\ell} \rho_{\ell}}{D t}\right)+p \frac{\partial \alpha_{\mathrm{g}} v_{\mathrm{g}}}{\partial x}+p \frac{\partial \alpha_{\ell} v_{\ell}}{\partial x} \\
=\left(\left(v_{\mathrm{g}}-v_{\ell}\right)\left(v_{\mathrm{i}}-\frac{1}{2}\left(v_{\mathrm{g}}+v_{\ell}\right)\right)+e_{\mathrm{g}}-e_{\ell}\right) \mathcal{K}\left(\mu_{\mathrm{g}}-\mu_{\ell}\right) .
\end{aligned}
$$

By the mass equations (2.1) and (2.2), it can be simplified to

$$
\alpha_{\mathrm{g}} \rho_{\mathrm{g}} T \frac{D_{\mathrm{g}} s_{\mathrm{g}}}{\partial t}+\alpha_{\ell} \rho_{\ell} T \frac{D_{\ell} s_{\ell}}{\partial t}=\left(\left(v_{\mathrm{g}}-v_{\ell}\right)\left(v_{\mathrm{i}}-\frac{1}{2}\left(v_{\mathrm{g}}+v_{\ell}\right)\right)+e_{\mathrm{g}}+\frac{p}{\rho_{\mathrm{g}}}-e_{\ell}-\frac{p}{\rho_{\ell}}\right) \mathcal{K}\left(\mu_{\mathrm{g}}-\mu_{\ell}\right),
$$

and using again the mass equations, we obtain the evolution equation for the mixture entropy

$$
T\left(\frac{\partial \alpha_{\mathrm{g}} \rho_{\mathrm{g}} s_{\mathrm{g}}}{\partial t}+\frac{\partial \alpha_{\ell} \rho_{\ell} s_{\ell}}{\partial t}+\frac{\partial \alpha_{\mathrm{g}} \rho_{\mathrm{g}} s_{\mathrm{g}} v_{\mathrm{g}}}{\partial x}+\frac{\partial \alpha_{\ell} \rho_{\ell} s_{\ell} v_{\ell}}{\partial x}\right)=\left(\left(v_{\mathrm{g}}-v_{\ell}\right)\left(v_{\mathrm{i}}-\frac{1}{2}\left(v_{\mathrm{g}}+v_{\ell}\right)\right)+\mu_{\mathrm{g}}-\mu_{\ell}\right) \mathcal{K}\left(\mu_{\mathrm{g}}-\mu_{\ell}\right)
$$

since the chemical potential can be expressed as in (2.7). Let us name the right-hand side as

$$
\mathcal{S}=\left(\left(v_{\mathrm{g}}-v_{\ell}\right)\left(v_{\mathrm{i}}-\frac{1}{2}\left(v_{\mathrm{g}}+v_{\ell}\right)\right)+\mu_{\mathrm{g}}-\mu_{\ell}\right) \mathcal{K}\left(\mu_{\mathrm{g}}-\mu_{\ell}\right),
$$

where we remind that $\mathcal{K}>0$. It may be written as

$$
\mathcal{S}=\mathcal{K}\left(w z+z^{2}\right)
$$

where

$$
\begin{aligned}
w & =\left(v_{\mathrm{g}}-v_{\ell}\right)\left(v_{\mathrm{i}}-\frac{1}{2}\left(v_{\mathrm{g}}+v_{\ell}\right)\right), \\
z & =\mu_{\mathrm{g}}-\mu_{\ell} .
\end{aligned}
$$


Now, the second law of thermodynamics imposes

$$
\mathcal{S} \geq 0 .
$$

For any given set of velocities, the entropy production attains its minimum when

$$
\frac{\mathrm{d} \mathcal{S}}{\mathrm{d} z}=\mathcal{K}(w+2 z)=0,
$$

hence when

$$
z=-\frac{w}{2} .
$$

Inserting this into (2.19), we obtain

$$
\mathcal{S}=-\mathcal{K} \frac{w^{2}}{4} .
$$

Thus, the second law of thermodynamics imposes

$$
w=0,
$$

which uniquely determines

$$
v_{\mathrm{i}}=\frac{1}{2}\left(v_{\mathrm{g}}+v_{\ell}\right) .
$$

We remark that the identical expression was proposed by [44] in the context of the model we here consider. Here we have presented a rigorous physical motivation for this expression.

\section{The FOUR-EQUATION MODEL}

We wish to derive a four-equation model from the above five-equation model, where we assume the phase change to be instantaneous. This is achieved by letting $\mathcal{K} \rightarrow \infty$ in the model (2.1)-(2.5). We then explicitly assume that $\mu_{\mathrm{g}}=\mu_{\ell}$, a relation that holds only in the two-phase region defined by the boiling point curve. Furthermore, in the limit $\alpha \rightarrow 0^{+}$, the velocity of the vanishing phase does not disappear from our definition $(2.27)$ of $v_{\mathrm{i}}$. Hence, for the purposes of this paper, we limit our analysis to the genuinely two-phase region $\alpha_{k} \in(0,1)$ and leave the study of transitions to one-phase flows to further works.

Since the repartition of the mass in the phases now is entirely governed by thermodynamics, we only need one mixture mass evolution equation, instead of one for each phase as in (2.1)-(2.2). We therefore sum (2.1) and (2.2) to give the mixture mass evolution equation of the four-equation model

$$
\frac{\partial\left(\alpha_{\mathrm{g}} \rho_{\mathrm{g}}+\alpha_{\ell} \rho_{\ell}\right)}{\partial t}+\frac{\partial\left(\alpha_{\mathrm{g}} \rho_{\mathrm{g}} v_{\mathrm{g}}+\alpha_{\ell} \rho_{\ell} v_{\ell}\right)}{\partial x}=0
$$

and specify $\mu_{\mathrm{g}}=\mu_{\ell}$. The remaining three other evolution equations of the four-equation model are the same as in the five-equation model (2.3)-(2.5). However, since $\mathcal{K} \rightarrow \infty$ and $\mu_{\mathrm{g}}=\mu_{\ell}, \mathcal{K}\left(\mu_{\mathrm{g}}-\mu_{\ell}\right)$ is an undefined limit. It needs to be substituted using the phase mass equations (2.1) and (2.2). This gives the model [40]

$$
\begin{gathered}
\frac{\partial\left(\alpha_{\mathrm{g}} \rho_{\mathrm{g}}+\alpha_{\ell} \rho_{\ell}\right)}{\partial t}+\frac{\partial\left(\alpha_{\mathrm{g}} \rho_{\mathrm{g}} v_{\mathrm{g}}+\alpha_{\ell} \rho_{\ell} v_{\ell}\right)}{\partial x}=0 \\
\frac{\partial \alpha_{\mathrm{g}} \rho_{\mathrm{g}} v_{\mathrm{g}}}{\partial t}+\frac{\partial \alpha_{\mathrm{g}} \rho_{\mathrm{g}} v_{\mathrm{g}}^{2}}{\partial x}+\alpha_{\mathrm{g}} \frac{\partial p}{\partial x}=\frac{v_{\mathrm{g}}+v_{\ell}}{2}\left(\frac{\partial \alpha_{\mathrm{g}} \rho_{\mathrm{g}}}{\partial t}+\frac{\partial \alpha_{\mathrm{g}} \rho_{\mathrm{g}} v_{\mathrm{g}}}{\partial x}\right), \\
\frac{\partial \alpha_{\ell} \rho_{\ell} v_{\ell}}{\partial t}+\frac{\partial \alpha_{\ell} \rho_{\ell} v_{\ell}^{2}}{\partial x}+\alpha_{\ell} \frac{\partial p}{\partial x}=\frac{v_{\mathrm{g}}+v_{\ell}}{2}\left(\frac{\partial \alpha_{\ell} \rho_{\ell}}{\partial t}+\frac{\partial \alpha_{\ell} \rho_{\ell} v_{\ell}}{\partial x}\right), \\
\frac{\partial\left(E_{\mathrm{g}}+E_{\ell}\right)}{\partial t}+\frac{\partial}{\partial x}\left(\left(E_{\mathrm{g}}+\alpha_{\mathrm{g}} p\right) v_{\mathrm{g}}+\left(E_{\ell}+\alpha_{\ell} p\right) v_{\ell}\right)=0 .
\end{gathered}
$$


Further, the internal energy equation becomes

$$
\frac{\partial}{\partial t}\left(\alpha_{\mathrm{g}} \rho_{\mathrm{g}} e_{\mathrm{g}}+\alpha_{\ell} \rho_{\ell} e_{\ell}\right)+\frac{\partial}{\partial x}\left(\alpha_{\mathrm{g}} \rho_{\mathrm{g}} e_{\mathrm{g}} v_{\mathrm{g}}+\alpha_{\ell} \rho_{\ell} e_{\ell} v_{\ell}\right)+p \frac{\partial \alpha_{\mathrm{g}} v_{\mathrm{g}}}{\partial x}+p \frac{\partial \alpha_{\ell} v_{\ell}}{\partial x}=0 .
$$

In the entropy equation (2.17), since $\mathcal{K}\left(\mu_{\mathrm{g}}-\mu_{\ell}\right)$ is finite, we have that $\mathcal{K}\left(\mu_{\mathrm{g}}-\mu_{\ell}\right)^{2} \rightarrow 0$. The entropy equation becomes

$$
\frac{\partial \alpha_{\mathrm{g}} \rho_{\mathrm{g}} s_{\mathrm{g}}}{\partial t}+\frac{\partial \alpha_{\ell} \rho_{\ell} s_{\ell}}{\partial t}+\frac{\partial \alpha_{\mathrm{g}} \rho_{\mathrm{g}} s_{\mathrm{g}} v_{\mathrm{g}}}{\partial x}+\frac{\partial \alpha_{\ell} \rho_{\ell} s_{\ell} v_{\ell}}{\partial x}=0
$$

Now, to be able to have the model in quasilinear form, we first need to express the time derivatives $\partial_{t} \alpha_{\mathrm{g}} \rho_{\mathrm{g}}$ and $\partial_{t} \alpha_{\ell} \rho_{\ell}$ in terms of spatial derivatives.

\subsection{Some differentials}

Some useful differentials can be derived from the assumptions of equilibrium.

Proposition 3.1. The differential of the pressure can be related to that of the temperature by

$$
\left(\frac{1}{\rho_{\mathrm{g}}}-\frac{1}{\rho_{\ell}}\right) \mathrm{d} p=\frac{L}{T} \mathrm{~d} T
$$

where

$$
L=e_{\mathrm{g}}+\frac{p}{\rho_{\mathrm{g}}}-e_{\ell}-\frac{p}{\rho_{\ell}}
$$

is the latent heat.

Proof. From the expression of the thermodynamic potential (2.7) and the fundamental thermodynamic relation (2.14), we obtain

$$
\mathrm{d} \mu_{k}=\frac{1}{\rho_{k}} \mathrm{~d} p-s_{k} \mathrm{~d} T .
$$

Since $\mu_{\mathrm{g}}=\mu_{\ell}$, we can write

$$
\left(\frac{1}{\rho_{\mathrm{g}}}-\frac{1}{\rho_{\ell}}\right) \mathrm{d} p=\left(s_{\mathrm{g}}-s_{\ell}\right) \mathrm{d} T .
$$

Remark that, with the Clapeyron equation, we can write

$$
s_{\mathrm{g}}-s_{\ell}=\frac{L}{T}
$$

Thus the differential becomes

$$
\left(\frac{1}{\rho_{\mathrm{g}}}-\frac{1}{\rho_{\ell}}\right) \mathrm{d} p=\frac{L}{T} \mathrm{~d} T .
$$

Then, we can obtain simplified entropy and internal energy differentials.

Proposition 3.2. For the phase $k$ being either the gas or the liquid phase, the entropy differential is

$$
\mathrm{d} s_{k}=-C_{p, k} \chi_{k} \mathrm{~d} p
$$

and the internal energy differential is

$$
\mathrm{d} e_{k}=\left(\frac{p}{\rho_{k}^{2} c_{k}^{2}} \Psi_{k}-T C_{p, k} \chi_{k}\right) \mathrm{d} p
$$


where

$$
\chi_{k}=\frac{\Gamma_{k}}{\rho_{k} c_{k}^{2}}+\frac{\rho_{\mathrm{g}}-\rho_{\ell}}{\rho_{\mathrm{g}} \rho_{\ell} L},
$$

and

$$
\Psi_{k}=1+\rho_{k} T C_{p, k} \Gamma_{k} \chi_{k},
$$

in which the first Grüneisen parameter is defined as

$$
\Gamma_{k} \equiv \frac{1}{\rho_{k}}\left(\frac{\partial p}{\partial e_{k}}\right)_{\rho_{k}} .
$$

Proof. An entropy differential may be found in [15]. For the gas phase, it reads

$$
\mathrm{d} s_{\mathrm{g}}=-\frac{\Gamma_{\mathrm{g}} C_{p, \mathrm{~g}}}{\rho_{\mathrm{g}} c_{\mathrm{g}}^{2}} \mathrm{~d} p+\frac{C_{p, \mathrm{~g}}}{T} \mathrm{~d} T,
$$

which with the help of (3.8) becomes

$$
\mathrm{d} s_{\mathrm{g}}=-C_{p, \mathrm{~g}}\left(\frac{\Gamma_{\mathrm{g}}}{\rho_{\mathrm{g}} c_{\mathrm{g}}^{2}}+\frac{\rho_{\mathrm{g}}-\rho_{\ell}}{\rho_{\mathrm{g}} \rho_{\ell} L}\right) \mathrm{d} p .
$$

To simplify the results, the shorthands (3.16) and (3.17) have been defined for expressions which repetitively appear in the present article. This gives the result (3.14).

On the other hand, an internal energy differential may be found in [17]. For the gas phase, it reads

$$
\begin{aligned}
\mathrm{d} e_{\mathrm{g}} & =\left(\frac{\partial e_{\mathrm{g}}}{\partial T}\right)_{p} \mathrm{~d} T+\left(\frac{\partial e_{\mathrm{g}}}{\partial p}\right)_{T} \mathrm{~d} p \\
& =C_{p, \mathrm{~g}}\left(1-\frac{\Gamma_{\mathrm{g}} p}{\rho_{\mathrm{g}} c_{\mathrm{g}}^{2}}\right) \mathrm{d} T+\left(\frac{p}{\rho_{\mathrm{g}}^{2} c_{\mathrm{g}}^{2}}-\frac{\Gamma_{\mathrm{g}} T}{\rho_{\mathrm{g}} c_{\mathrm{g}}^{2}} C_{p, \mathrm{~g}}\left(1-\frac{\Gamma_{\mathrm{g}} p}{\rho_{\mathrm{g}} c_{\mathrm{g}}^{2}}\right)\right) \mathrm{d} p
\end{aligned}
$$

which can be written through (3.8) as

$$
\mathrm{d} e_{\mathrm{g}}=\frac{1}{\rho_{\mathrm{g}} c_{\mathrm{g}}^{2}}\left(\frac{p}{\rho_{\mathrm{g}}}-T C_{p, \mathrm{~g}}\left(\rho_{\mathrm{g}} c_{\mathrm{g}}^{2}-\Gamma_{\mathrm{g}} p\right)\left(\frac{\rho_{\mathrm{g}}-\rho_{\ell}}{\rho_{\mathrm{g}} \rho_{\ell} L}+\frac{\Gamma_{\mathrm{g}}}{\rho_{\mathrm{g}} c_{\mathrm{g}}^{2}}\right)\right) \mathrm{d} p .
$$

Using the shorthands (3.16) and (3.17), this gives the result (3.15). Note that this expression may be written, through (3.14), as

$$
\mathrm{d} e_{\mathrm{g}}=\frac{p}{\rho_{\mathrm{g}}^{2} c_{\mathrm{g}}^{2}} \Psi_{\mathrm{g}} \mathrm{d} p+T \mathrm{~d} s_{\mathrm{g}} .
$$

The counterpart for the liquid phase of these differentials is found by symmetry of the phases.

\subsection{Treatment of the time derivatives}

The momentum equations (3.3) and (3.4) contain time derivatives, which we wish to convert to spatial derivatives.

Proposition 3.3. The relaxed gas-phase mass equation may be written as

$$
\frac{\partial \alpha_{\mathrm{g}} \rho_{\mathrm{g}}}{\partial t}+\frac{\partial \alpha_{\mathrm{g}} \rho_{\mathrm{g}} v_{\mathrm{g}}}{\partial x}=-\mathcal{P} \frac{\partial p}{\partial x}-\mathcal{V}\left(\frac{\partial \alpha_{\mathrm{g}} v_{\mathrm{g}}}{\partial x}+\frac{\partial \alpha_{\ell} v_{\ell}}{\partial x}\right),
$$


where

$$
\begin{aligned}
& \mathcal{V}=\frac{T\left(\alpha_{\mathrm{g}} \rho_{\mathrm{g}} C_{p, \mathrm{~g}} \chi_{\mathrm{g}}+\alpha_{\ell} \rho_{\ell} C_{p, \ell} \chi_{\ell}\right)}{L\left(\frac{\alpha_{\mathrm{g}}}{\rho_{\mathrm{g}} c_{\mathrm{g}}^{2}} \Psi_{\mathrm{g}}+\frac{\alpha_{\ell}}{\rho_{\ell} c_{\ell}^{2}} \Psi_{\ell}\right)+T\left(\alpha_{\mathrm{g}} \rho_{\mathrm{g}} C_{p, \mathrm{~g}} \chi_{\mathrm{g}}+\alpha_{\ell} \rho_{\ell} C_{p, \ell} \chi_{\ell}\right) \frac{\rho_{\mathrm{g}}-\rho_{\ell}}{\rho_{\mathrm{g}} \rho_{\ell}}}, \\
& \mathcal{P}=\frac{\alpha_{\mathrm{g}} \alpha_{\ell} T\left(v_{\mathrm{g}}-v_{\ell}\right)\left(\rho_{\ell} C_{p, \ell} \chi_{\ell} \frac{\Psi_{\mathrm{g}}}{\rho_{\mathrm{g}} c_{\mathrm{g}}^{2}}-\rho_{\mathrm{g}} C_{p, \mathrm{~g}} \chi_{\mathrm{g}} \frac{\Psi_{\ell}}{\rho_{\ell} c_{\ell}^{2}}\right)}{L\left(\frac{\alpha_{\mathrm{g}}}{\rho_{\mathrm{g}} c_{\mathrm{g}}^{2}} \Psi_{\mathrm{g}}+\frac{\alpha_{\ell}}{\rho_{\ell} c_{\ell}^{2}} \Psi_{\ell}\right)+T\left(\alpha_{\mathrm{g}} \rho_{\mathrm{g}} C_{p, \mathrm{~g}} \chi_{\mathrm{g}}+\alpha_{\ell} \rho_{\ell} C_{p, \ell} \chi_{\ell}\right) \frac{\rho_{\mathrm{g}}-\rho_{\ell}}{\rho_{\mathrm{g}} \rho_{\ell}}} .
\end{aligned}
$$

This expression can be substituted in the momentum equation for the gas phase (3.3), thus eliminating the time derivatives. For the liquid phase, the relaxed mass equation reads

$$
\frac{\partial \alpha_{\ell} \rho_{\ell}}{\partial t}+\frac{\partial \alpha_{\ell} \rho_{\ell} v_{\ell}}{\partial x}=\mathcal{P} \frac{\partial p}{\partial x}+\mathcal{V}\left(\frac{\partial \alpha_{\mathrm{g}} v_{\mathrm{g}}}{\partial x}+\frac{\partial \alpha_{\ell} v_{\ell}}{\partial x}\right)
$$

Proof. From the differentials (3.14) and (3.15) as well as the mixture mass equation (3.2), internal energy equation (3.6) and entropy equation (3.7), we are able to find three relations between $\partial_{t} p, \partial_{t} \alpha_{\mathrm{g}} \rho_{\mathrm{g}}$ and $\partial_{t} \alpha_{\ell} \rho_{\ell}$ and spatial derivatives. Therefore we can find an expression for each of the time derivatives.

The first relation is the mass equation (3.2)

$$
\frac{\partial\left(\alpha_{\mathrm{g}} \rho_{\mathrm{g}}+\alpha_{\ell} \rho_{\ell}\right)}{\partial t}+\frac{\partial\left(\alpha_{\mathrm{g}} \rho_{\mathrm{g}} v_{\mathrm{g}}+\alpha_{\ell} \rho_{\ell} v_{\ell}\right)}{\partial x}=0
$$

Then, the derivatives are expanded in the entropy equation (3.7). The derivatives $\partial_{t} s_{k}$ and $\partial_{x} s_{k}$ are subsequently substituted using the entropy differential (3.14) to obtain a second relation

$$
\begin{aligned}
-\left(\alpha_{\mathrm{g}} \rho_{\mathrm{g}} C_{p, \mathrm{~g}} \chi_{\mathrm{g}}+\alpha_{\ell} \rho_{\ell} C_{p, \ell} \chi_{\ell}\right) \frac{\partial p}{\partial t}-\left(\alpha_{\mathrm{g}} \rho_{\mathrm{g}} C_{p, \mathrm{~g}} \chi_{\mathrm{g}} v_{\mathrm{g}}\right. & \left.+\alpha_{\ell} \rho_{\ell} C_{p, \ell} \chi_{\ell} v_{\ell}\right) \frac{\partial p}{\partial x} \\
& +s_{\mathrm{g}} \frac{\partial \alpha_{\mathrm{g}} \rho_{\mathrm{g}}}{\partial t}+s_{\ell} \frac{\partial \alpha_{\ell} \rho_{\ell}}{\partial t}+s_{\mathrm{g}} \frac{\partial \alpha_{\mathrm{g}} \rho_{\mathrm{g}} v_{\mathrm{g}}}{\partial x}+s_{\ell} \frac{\partial \alpha_{\ell} \rho_{\ell} v_{\ell}}{\partial x}=0 .
\end{aligned}
$$

Finally, the same treatment is applied to the internal energy equation (3.6) with the differential (3.15), which gives a third relation

$$
\begin{aligned}
&\left(\alpha_{\mathrm{g}} \rho_{\mathrm{g}}\left(\frac{p}{\rho_{\mathrm{g}}^{2} c_{\mathrm{g}}^{2}} \Psi_{\mathrm{g}}-T C_{p, \mathrm{~g}} \chi_{\mathrm{g}}\right)\right.\left.+\alpha_{\ell} \rho_{\ell}\left(\frac{p}{\rho_{\ell}^{2} c_{\ell}^{2}} \Psi_{\ell}-T C_{p, \ell} \chi_{\ell}\right)\right) \frac{\partial p}{\partial t} \\
&+\left(\alpha_{\mathrm{g}} \rho_{\mathrm{g}}\left(\frac{p}{\rho_{\mathrm{g}}^{2} c_{\mathrm{g}}^{2}} \Psi_{\mathrm{g}}-T C_{p, \mathrm{~g}} \chi_{\mathrm{g}}\right) v_{\mathrm{g}}+\alpha_{\ell} \rho_{\ell}\left(\frac{p}{\rho_{\ell}^{2} c_{\ell}^{2}} \Psi_{\ell}-T C_{p, \ell} \chi_{\ell}\right) v_{\ell}\right) \frac{\partial p}{\partial x} \\
& \quad+e_{\mathrm{g}} \frac{\partial \alpha_{\mathrm{g}} \rho_{\mathrm{g}}}{\partial t}+e_{\ell} \frac{\partial \alpha_{\ell} \rho_{\ell}}{\partial t}+e_{\mathrm{g}} \frac{\partial \alpha_{\mathrm{g}} \rho_{\mathrm{g}} v_{\mathrm{g}}}{\partial x}+e_{\ell} \frac{\partial \alpha_{\ell} \rho_{\ell} v_{\ell}}{\partial x}+p \frac{\partial \alpha_{\mathrm{g}} v_{\mathrm{g}}}{\partial x}+p \frac{\partial \alpha_{\ell} v_{\ell}}{\partial x}=0
\end{aligned}
$$

Solving these three relations, we obtain the relaxed gas-phase mass equation (3.24). To find the equation for the liquid phase, we remark that the mixture mass equation (3.2) gives

$$
\frac{\partial \alpha_{\ell} \rho_{\ell}}{\partial t}+\frac{\partial \alpha_{\ell} \rho_{\ell} v_{\ell}}{\partial x}=-\frac{\partial \alpha_{\mathrm{g}} \rho_{\mathrm{g}}}{\partial t}-\frac{\partial \alpha_{\mathrm{g}} \rho_{\mathrm{g}} v_{\mathrm{g}}}{\partial x}
$$

which gives the result through (3.24). 


\subsection{Regularising term}

As with the six- and five-equation two-fluid models, we expect the present four-equation model not to be hyperbolic when the gas and liquid velocities are different from each other $[16,19,45]$. The eigenvalues associated with the volume-fraction waves are expected to be complex. For the purposes of this paper, we consider including a regularising term similar to the interfacial-pressure regularising term for the six-equation two-fluid model $[6,10,12,13,35,46]$. It consists in applying a pressure difference $\Delta p$ between the two phases. The momentum equations are transformed into

$$
\frac{\partial \alpha_{\mathrm{g}} \rho_{\mathrm{g}} v_{\mathrm{g}}}{\partial t}+\frac{\partial \alpha_{\mathrm{g}} \rho_{\mathrm{g}} v_{\mathrm{g}}^{2}}{\partial x}+\alpha_{\mathrm{g}} \frac{\partial p}{\partial x}+\Delta p \frac{\partial \alpha_{\mathrm{g}}}{\partial x}=\frac{v_{\mathrm{g}}+v_{\ell}}{2}\left(\frac{\partial \alpha_{\mathrm{g}} \rho_{\mathrm{g}}}{\partial t}+\frac{\partial \alpha_{\mathrm{g}} \rho_{\mathrm{g}} v_{\mathrm{g}}}{\partial x}\right)
$$

and

$$
\frac{\partial \alpha_{\ell} \rho_{\ell} v_{\ell}}{\partial t}+\frac{\partial \alpha_{\ell} \rho_{\ell} v_{\ell}^{2}}{\partial x}+\alpha_{\ell} \frac{\partial p}{\partial x}+\Delta p \frac{\partial \alpha_{\ell}}{\partial x}=\frac{v_{\mathrm{g}}+v_{\ell}}{2}\left(\frac{\partial \alpha_{\ell} \rho_{\ell}}{\partial t}+\frac{\partial \alpha_{\ell} \rho_{\ell} v_{\ell}}{\partial x}\right)
$$

while the mass and energy equations are not modified. The effect of this term will be analyzed in Section 6 .

\subsection{Expression of the model}

As a result of the present section, the four-equation model (3.2)-(3.5) can be written, using (3.24), (3.27), (3.32) and (3.33), in the following form

$$
\begin{gathered}
\frac{\partial\left(\alpha_{\mathrm{g}} \rho_{\mathrm{g}}+\alpha_{\ell} \rho_{\ell}\right)}{\partial t}+\frac{\partial\left(\alpha_{\mathrm{g}} \rho_{\mathrm{g}} v_{\mathrm{g}}+\alpha_{\ell} \rho_{\ell} v_{\ell}\right)}{\partial x}=0 \\
\frac{\partial \alpha_{\mathrm{g}} \rho_{\mathrm{g}} v_{\mathrm{g}}}{\partial t}+\frac{\partial \alpha_{\mathrm{g}} \rho_{\mathrm{g}} v_{\mathrm{g}}^{2}}{\partial x}+\left(\alpha_{\mathrm{g}}+\frac{v_{\mathrm{g}}+v_{\ell}}{2} \mathcal{P}\right) \frac{\partial p}{\partial x}+\frac{v_{\mathrm{g}}+v_{\ell}}{2} \mathcal{V} \frac{\partial\left(\alpha_{\mathrm{g}} v_{\mathrm{g}}+\alpha_{\ell} v_{\ell}\right)}{\partial x}+\Delta p \frac{\partial \alpha_{\mathrm{g}}}{\partial x}=0 \\
\frac{\partial \alpha_{\ell} \rho_{\ell} v_{\ell}}{\partial t}+\frac{\partial \alpha_{\ell} \rho_{\ell} v_{\ell}^{2}}{\partial x}+\left(\alpha_{\ell}-\frac{v_{\mathrm{g}}+v_{\ell}}{2} \mathcal{P}\right) \frac{\partial p}{\partial x}-\frac{v_{\mathrm{g}}+v_{\ell}}{2} \mathcal{V} \frac{\partial\left(\alpha_{\mathrm{g}} v_{\mathrm{g}}+\alpha_{\ell} v_{\ell}\right)}{\partial x}+\Delta p \frac{\partial \alpha_{\ell}}{\partial x}=0 \\
\frac{\partial\left(E_{\mathrm{g}}+E_{\ell}\right)}{\partial t}+\frac{\partial}{\partial x}\left(\left(E_{\mathrm{g}}+\alpha_{\mathrm{g}} p\right) v_{\mathrm{g}}+\left(E_{\ell}+\alpha_{\ell} p\right) v_{\ell}\right)=0
\end{gathered}
$$

This model has been previously considered in $[40,44]$. The original contribution of this section has been to express the model in terms of spatial derivatives only; this will prove useful when we now turn to deriving the quasilinear formulation.

\section{QUASILINEAR FORM}

We wish to write the model in quasilinear form

$$
\frac{\partial \boldsymbol{U}}{\partial t}+\boldsymbol{A}(\boldsymbol{U}) \frac{\partial \boldsymbol{U}}{\partial x}=0
$$

where the vector of variables $\boldsymbol{U}$ is defined as

$$
\boldsymbol{U}=\left(\begin{array}{c}
\alpha_{\mathrm{g}} \rho_{\mathrm{g}}+\alpha_{\ell} \rho_{\ell} \\
\alpha_{\mathrm{g}} \rho_{\mathrm{g}} v_{\mathrm{g}} \\
\alpha_{\ell} \rho_{\ell} v_{\ell} \\
E_{\mathrm{g}}+E_{\ell}
\end{array}\right)
$$


The matrix $\boldsymbol{A}(\boldsymbol{U})$ is the Jacobian of the flux. The flux is split into a conservative and a non-conservative part, such that the system can be written as

$$
\frac{\partial \boldsymbol{U}}{\partial t}+\frac{\partial \boldsymbol{F}_{c}(\boldsymbol{U})}{\partial x}+\boldsymbol{B}(\boldsymbol{U}) \frac{\partial \boldsymbol{W}(\boldsymbol{U})}{\partial x}=0
$$

where the conservative flux is

$$
\boldsymbol{F}_{c}(\boldsymbol{U})=\left(\begin{array}{c}
\alpha_{\mathrm{g}} \rho_{\mathrm{g}} v_{\mathrm{g}}+\alpha_{\ell} \rho_{\ell} v_{\ell} \\
\alpha_{\mathrm{g}} \rho_{\mathrm{g}} v_{\mathrm{g}}^{2} \\
\alpha_{\ell} \rho_{\ell} v_{\ell}^{2} \\
\left(E_{\mathrm{g}}+\alpha_{\mathrm{g}} p\right) v_{\mathrm{g}}+\left(E_{\ell}+\alpha_{\ell} p\right) v_{\ell}
\end{array}\right)
$$

while the non-conservative contributions are

$$
\boldsymbol{B}(\boldsymbol{U})=\left(\begin{array}{ccc}
0 & 0 & 0 \\
\alpha_{\mathrm{g}}+\frac{v_{\mathrm{g}}+v_{\ell}}{2} \mathcal{P} & \frac{v_{\mathrm{g}}+v_{\ell}}{2} \mathcal{V} & \Delta p \\
\alpha_{\ell}-\frac{v_{\mathrm{g}}+v_{\ell}}{2} \mathcal{P} & -\frac{v_{\mathrm{g}}+v_{\ell}}{2} \mathcal{V} & -\Delta p \\
0 & 0 & 0
\end{array}\right) \quad \text { and } \quad \boldsymbol{W}=\left(\begin{array}{c}
p \\
\alpha_{\mathrm{g}} v_{\mathrm{g}}+\alpha_{\ell} v_{\ell} \\
\alpha_{\mathrm{g}}
\end{array}\right)
$$

\subsection{Some differentials}

In order to write the Jacobian of the fluxes, we need to express the differentials of some variables in terms of the differential of the components of the variable vector $\boldsymbol{U}$. We will find them with the help of the fundamental relation of thermodynamics (2.14) as well as the differentials of the components of the vector $\boldsymbol{U}$. First, we will express all the differentials in terms of the differential of the gas density. Then, the other differentials will follow.

Proposition 4.1. The density differential may be expressed in terms of the differentials of the variable-vector components $u_{i}$ as

$$
\mathrm{d} \rho_{\mathrm{g}}=\frac{1}{\Phi} \frac{\Psi_{\mathrm{g}}}{c_{\mathrm{g}}^{2}}\left(\frac{\mathcal{E}}{\rho_{\mathrm{g}}-\rho_{\ell}} \mathrm{d} u_{1}-v_{\mathrm{g}} \mathrm{d} u_{2}-v_{\ell} \mathrm{d} u_{3}+\mathrm{d} u_{4}\right)
$$

where we have used the following shorthands

$$
\begin{aligned}
\Phi=\alpha_{\mathrm{g}} \frac{p}{\rho_{\mathrm{g}} c_{\mathrm{g}}^{2}} \Psi_{\mathrm{g}}+\alpha_{\ell} \frac{p}{\rho_{\ell} c_{\ell}^{2}} \Psi_{\ell}-\left(\alpha_{\mathrm{g}} \rho_{\mathrm{g}} T C_{p, \mathrm{~g}} \chi_{\mathrm{g}}\right. & \left.\alpha_{\ell} \rho_{\ell} T C_{p, \ell} \chi_{\ell}\right) \\
& +\frac{1}{\rho_{\mathrm{g}}-\rho_{\ell}}\left(-e_{\mathrm{g}}+\frac{1}{2} v_{\mathrm{g}}^{2}+e_{\ell}-\frac{1}{2} v_{\ell}^{2}\right)\left(\alpha_{\mathrm{g}} \rho_{\ell} \frac{\Psi_{\mathrm{g}}}{c_{\mathrm{g}}^{2}}+\alpha_{\ell} \rho_{\mathrm{g}} \frac{\Psi_{\ell}}{c_{\ell}^{2}}\right)
\end{aligned}
$$

and

$$
\mathcal{E}=-\rho_{\mathrm{g}}\left(e_{\mathrm{g}}-\frac{1}{2} v_{\mathrm{g}}^{2}\right)+\rho_{\ell}\left(e_{\ell}-\frac{1}{2} v_{\ell}^{2}\right)
$$

Proof. We recall from the previous section the differential (3.23)

$$
\mathrm{d} e_{\mathrm{g}}=\frac{p}{\rho_{\mathrm{g}}^{2} c_{\mathrm{g}}^{2}} \Psi_{\mathrm{g}} \mathrm{d} p+T \mathrm{~d} s_{\mathrm{g}}
$$

By identification with the fundamental thermodynamic relation (2.14), we can deduce

$$
\Psi_{\mathrm{g}} \mathrm{d} p=c_{\mathrm{g}}^{2} \mathrm{~d} \rho_{\mathrm{g}}
$$


and using the relation between pressure and temperature differentials (3.8), we obtain

$$
-\Psi_{\mathrm{g}} \frac{\rho_{\mathrm{g}} \rho_{\ell} L}{T\left(\rho_{\mathrm{g}}-\rho_{\ell}\right)} \mathrm{d} T=c_{\mathrm{g}}^{2} \mathrm{~d} \rho_{\mathrm{g}} .
$$

Now, we write the differential of the thermodynamic potentials for both phases in terms of their respective density differentials, using (4.10) and (4.11)

$$
\begin{aligned}
& \mathrm{d} \mu_{\mathrm{g}}=\frac{1}{\rho_{\mathrm{g}}} \mathrm{d} p-s_{\mathrm{g}} \mathrm{d} T=\frac{1}{\rho_{\mathrm{g}}} \frac{c_{\mathrm{g}}^{2}}{\Psi_{\mathrm{g}}} \mathrm{d} \rho_{\mathrm{g}}+s_{\mathrm{g}} \frac{c_{\mathrm{g}}^{2}}{\Psi_{\mathrm{g}}} \frac{T\left(\rho_{\mathrm{g}}-\rho_{\ell}\right)}{\rho_{\mathrm{g}} \rho_{\ell} L} \mathrm{~d} \rho_{\mathrm{g}} \\
& \mathrm{d} \mu_{\ell}=\frac{1}{\rho_{\ell}} \mathrm{d} p-s_{\ell} \mathrm{d} T=\frac{1}{\rho_{\ell}} \frac{c_{\ell}^{2}}{\Psi_{\ell}} \mathrm{d} \rho_{\ell}+s_{\ell} \frac{c_{\ell}^{2}}{\Psi_{\ell}} \frac{T\left(\rho_{\mathrm{g}}-\rho_{\ell}\right)}{\rho_{\mathrm{g}} \rho_{\ell} L} \mathrm{~d} \rho_{\ell}
\end{aligned}
$$

and equate them, using the assumption of chemical equilibrium. Implicitly, we also use the mechanical and thermal equilibrium assumptions, since we have expressed the pressure and temperature differentials in terms of the gas as well as of the liquid phase variables. This gives a relation between the density differentials:

$$
\frac{c_{\mathrm{g}}^{2}}{\Psi_{\mathrm{g}}} \mathrm{d} \rho_{\mathrm{g}}=\frac{c_{\ell}^{2}}{\Psi_{\ell}} \mathrm{d} \rho_{\ell} .
$$

Next, we need a relation for the energy differentials. For the gas phase, we find it using the differential of $p\left(\rho_{\mathrm{g}}, e_{\mathrm{g}}\right)$

$$
\mathrm{d} p=\left(c_{\mathrm{g}}^{2}-\Gamma_{\mathrm{g}} \frac{p}{\rho_{\mathrm{g}}}\right) \mathrm{d} \rho_{\mathrm{g}}+\Gamma_{\mathrm{g}} \rho_{\mathrm{g}} \mathrm{d} e_{\mathrm{g}},
$$

where $\mathrm{d} p$ is replaced using (3.15). After simplification, we obtain

$$
\frac{\Psi_{\mathrm{g}}}{\left(\frac{p}{\rho_{\mathrm{g}}^{2} c_{\mathrm{g}}^{2}} \Psi_{\mathrm{g}}-T C_{p, \mathrm{~g}} \chi_{\mathrm{g}}\right)} \mathrm{d} e_{\mathrm{g}}=c_{\mathrm{g}}^{2} \mathrm{~d} \rho_{\mathrm{g}} .
$$

For the liquid phase, we first use the phase symmetry to obtain

$$
\frac{\Psi_{\ell}}{\left(\frac{p}{\rho_{\ell}^{2} c_{\ell}^{2}} \Psi_{\ell}-T C_{p, \ell} \chi_{\ell}\right)} \mathrm{d} e_{\ell}=c_{\ell}^{2} \mathrm{~d} \rho_{\ell},
$$

and then replace the liquid density differential using (4.14)

$$
\frac{1}{\left(\frac{p}{\rho_{\ell}^{2} c_{\ell}^{2}} \Psi_{\ell}-T C_{p, \ell} \chi_{\ell}\right)} \mathrm{d} e_{\ell}=\frac{c_{\mathrm{g}}^{2}}{\Psi_{\mathrm{g}}} \mathrm{d} \rho_{\mathrm{g}} .
$$

Further, we seek an expression for the differential of the volume fraction. From the differential of the first component of the vector $\boldsymbol{U}$, we have

$$
\mathrm{d} u_{1}=\alpha_{\mathrm{g}} \mathrm{d} \rho_{\mathrm{g}}+\alpha_{\ell} \mathrm{d} \rho_{\ell}+\left(\rho_{\mathrm{g}}-\rho_{\ell}\right) \mathrm{d} \alpha_{\mathrm{g}},
$$

where $\rho_{\ell}$ is eliminated using the differential (4.14)

$$
\left(\rho_{\mathrm{g}}-\rho_{\ell}\right) \mathrm{d} \alpha_{\mathrm{g}}=\mathrm{d} u_{1}-\left(\alpha_{\mathrm{g}}+\alpha_{\ell} \frac{c_{\mathrm{g}}^{2} \Psi_{\ell}}{c_{\ell}^{2} \Psi_{\mathrm{g}}}\right) \mathrm{d} \rho_{\mathrm{g}} .
$$


Finally, we would like to find an expression for the velocity differentials. For the gas phase, we start from the differential of the second component of the vector $\boldsymbol{U}$

$$
\mathrm{d} u_{2}=\mathrm{d}\left(\alpha_{\mathrm{g}} \rho_{\mathrm{g}} v_{\mathrm{g}}\right)=\alpha_{\mathrm{g}} \rho_{\mathrm{g}} \mathrm{d} v_{\mathrm{g}}+\alpha_{\mathrm{g}} v_{\mathrm{g}} \mathrm{d} \rho_{\mathrm{g}}+\rho_{\mathrm{g}} v_{\mathrm{g}} \mathrm{d} \alpha_{\mathrm{g}},
$$

where $\mathrm{d} \alpha_{\mathrm{g}}$ is replaced using (4.20) to obtain

$$
\alpha_{\mathrm{g}} \rho_{\mathrm{g}} \mathrm{d} v_{\mathrm{g}}=-\frac{\rho_{\mathrm{g}} v_{\mathrm{g}}}{\rho_{\mathrm{g}}-\rho_{\ell}} \mathrm{d} u_{1}+\mathrm{d} u_{2}+\frac{v_{\mathrm{g}}}{\rho_{\mathrm{g}}-\rho_{\ell}}\left(\alpha_{\mathrm{g}} \rho_{\ell}+\alpha_{\ell} \rho_{\mathrm{g}} \frac{c_{\mathrm{g}}^{2} \Psi_{\ell}}{c_{\ell}^{2} \Psi_{\mathrm{g}}}\right) \mathrm{d} \rho_{\mathrm{g}} .
$$

By phase symmetry, we deduce that

$$
\alpha_{\ell} \rho_{\ell} \mathrm{d} v_{\ell}=\frac{\rho_{\ell} v_{\ell}}{\rho_{\mathrm{g}}-\rho_{\ell}} \mathrm{d} u_{1}+\mathrm{d} u_{3}-\frac{v_{\ell}}{\rho_{\mathrm{g}}-\rho_{\ell}}\left(\alpha_{\ell} \rho_{\mathrm{g}}+\alpha_{\mathrm{g}} \rho_{\ell} \frac{c_{\ell}^{2} \Psi_{\mathrm{g}}}{c_{\mathrm{g}}^{2} \Psi_{\ell}}\right) \mathrm{d} \rho_{\ell} .
$$

In order to express it in terms of the differential for the gas density, we use (4.14) to obtain

$$
\alpha_{\ell} \rho_{\ell} \mathrm{d} v_{\ell}=\frac{\rho_{\ell} v_{\ell}}{\rho_{\mathrm{g}}-\rho_{\ell}} \mathrm{d} u_{1}+\mathrm{d} u_{3}-\frac{v_{\ell}}{\rho_{\mathrm{g}}-\rho_{\ell}}\left(\alpha_{\mathrm{g}} \rho_{\ell}+\alpha_{\ell} \rho_{\mathrm{g}} \frac{c_{\mathrm{g}}^{2} \Psi_{\ell}}{c_{\ell}^{2} \Psi_{\mathrm{g}}}\right) \mathrm{d} \rho_{\mathrm{g}} .
$$

Now, using the differential of the mixture internal energy, we are able to deduce a differential for the gas density $\mathrm{d} \rho_{\mathrm{g}}$. We have that

$$
\mathrm{d}\left(\alpha_{\mathrm{g}} \rho_{\mathrm{g}} e_{\mathrm{g}}\right)+\mathrm{d}\left(\alpha_{\ell} \rho_{\ell} e_{\ell}\right)=\mathrm{d} u_{4}-\frac{v_{\mathrm{g}}}{2} \mathrm{~d} u_{2}-\frac{v_{\ell}}{2} \mathrm{~d} u_{3}-\frac{1}{2} \alpha_{\mathrm{g}} \rho_{\mathrm{g}} v_{\mathrm{g}} \mathrm{d} v_{\mathrm{g}}-\frac{1}{2} \alpha_{\ell} \rho_{\ell} v_{\ell} \mathrm{d} v_{\ell} .
$$

After having replaced all the differentials using the expressions (4.14), (4.16), (4.18), (4.20), (4.22) and (4.24) previously derived, we obtain the density differential (4.6).

All the other differentials now follow. The differential of the volume fraction follows from (4.20) in which $\mathrm{d} \rho_{\mathrm{g}}$ is replaced using (4.6)

$$
\mathrm{d} \alpha_{\mathrm{g}}=\frac{1}{\rho_{\mathrm{g}}-\rho_{\ell}} \mathrm{d} u_{1}-\frac{1}{\rho_{\mathrm{g}}-\rho_{\ell}} \frac{1}{\Phi}\left(\alpha_{\mathrm{g}} \frac{\Psi_{\mathrm{g}}}{c_{\mathrm{g}}^{2}}+\alpha_{\ell} \frac{\Psi_{\ell}}{c_{\ell}^{2}}\right)\left(\frac{\mathcal{E}}{\rho_{\mathrm{g}}-\rho_{\ell}} \mathrm{d} u_{1}-v_{\mathrm{g}} \mathrm{d} u_{2}-v_{\ell} \mathrm{d} u_{3}+\mathrm{d} u_{4}\right) .
$$

The differential of the pressure follows from (4.10)

$$
\mathrm{d} p=\frac{1}{\Phi}\left(\frac{\mathcal{E}}{\rho_{\mathrm{g}}-\rho_{\ell}} \mathrm{d} u_{1}-v_{\mathrm{g}} \mathrm{d} u_{2}-v_{\ell} \mathrm{d} u_{3}+\mathrm{d} u_{4}\right)
$$

The differential of the liquid density follows from (4.14)

$$
\mathrm{d} \rho_{\ell}=\frac{1}{\Phi} \frac{\Psi_{\ell}}{c_{\ell}^{2}}\left(\frac{\mathcal{E}}{\rho_{\mathrm{g}}-\rho_{\ell}} \mathrm{d} u_{1}-v_{\mathrm{g}} \mathrm{d} u_{2}-v_{\ell} \mathrm{d} u_{3}+\mathrm{d} u_{4}\right) .
$$

The differentials of the internal energies follow from (4.16) and (4.18)

$$
\begin{aligned}
\mathrm{d} e_{\mathrm{g}} & =\frac{1}{\Phi}\left(\frac{p}{\rho_{\mathrm{g}}^{2} c_{\mathrm{g}}^{2}} \Psi_{\mathrm{g}}-T C_{p, \mathrm{~g}} \chi_{\mathrm{g}}\right)\left(\frac{\mathcal{E}}{\rho_{\mathrm{g}}-\rho_{\ell}} \mathrm{d} u_{1}-v_{\mathrm{g}} \mathrm{d} u_{2}-v_{\ell} \mathrm{d} u_{3}+\mathrm{d} u_{4}\right), \\
\mathrm{d} e_{\ell} & =\frac{1}{\Phi}\left(\frac{p}{\rho_{\ell}^{2} c_{\ell}^{2}} \Psi_{\ell}-T C_{p, \ell} \chi_{\ell}\right)\left(\frac{\mathcal{E}}{\rho_{\mathrm{g}}-\rho_{\ell}} \mathrm{d} u_{1}-v_{\mathrm{g}} \mathrm{d} u_{2}-v_{\ell} \mathrm{d} u_{3}+\mathrm{d} u_{4}\right) .
\end{aligned}
$$


The differentials of the velocities follow from (4.22) and (4.24)

$$
\begin{aligned}
\alpha_{\mathrm{g}} \rho_{\mathrm{g}} \mathrm{d} v_{\mathrm{g}}= & -\frac{\rho_{\mathrm{g}} v_{\mathrm{g}}}{\rho_{\mathrm{g}}-\rho_{\ell}} \mathrm{d} u_{1}+\mathrm{d} u_{2}+\frac{1}{\Phi} \frac{v_{\mathrm{g}}}{\rho_{\mathrm{g}}-\rho_{\ell}} \\
& \times\left(\alpha_{\mathrm{g}} \rho_{\ell} \frac{\Psi_{\mathrm{g}}}{c_{\mathrm{g}}^{2}}+\alpha_{\ell} \rho_{\mathrm{g}} \frac{\Psi_{\ell}}{c_{\ell}^{2}}\right)\left(\frac{\mathcal{E}}{\rho_{\mathrm{g}}-\rho_{\ell}} \mathrm{d} u_{1}-v_{\mathrm{g}} \mathrm{d} u_{2}-v_{\ell} \mathrm{d} u_{3}+\mathrm{d} u_{4}\right), \\
\alpha_{\ell} \rho_{\ell} \mathrm{d} v_{\ell}= & \frac{\rho_{\ell} v_{\ell}}{\rho_{\mathrm{g}}-\rho_{\ell}} \mathrm{d} u_{1}+\mathrm{d} u_{3}-\frac{1}{\Phi} \frac{v_{\ell}}{\rho_{\mathrm{g}}-\rho_{\ell}} \\
& \times\left(\alpha_{\mathrm{g}} \rho_{\ell} \frac{\Psi_{\mathrm{g}}}{c_{\mathrm{g}}^{2}}+\alpha_{\ell} \rho_{\mathrm{g}} \frac{\Psi_{\ell}}{c_{\ell}^{2}}\right)\left(\frac{\mathcal{E}}{\rho_{\mathrm{g}}-\rho_{\ell}} \mathrm{d} u_{1}-v_{\mathrm{g}} \mathrm{d} u_{2}-v_{\ell} \mathrm{d} u_{3}+\mathrm{d} u_{4}\right) .
\end{aligned}
$$

\subsection{Jacobian of the fluxes}

We are now able to derive the Jacobian of the conservative fluxes $\boldsymbol{F}_{c}(\boldsymbol{U})(4.4)$ and of the vector $\boldsymbol{W}(\boldsymbol{U})$ in the non-conservative fluxes (4.5). To do so, we express the differentials of the components of the vectors $\boldsymbol{F}_{c}(\boldsymbol{U})$ and $\boldsymbol{W}(\boldsymbol{U})$ in terms of the differentials of the components of $\boldsymbol{U}$. First, we simply have

$$
\mathrm{d} f_{1}=\mathrm{d}\left(\alpha_{\mathrm{g}} \rho_{\mathrm{g}} v_{\mathrm{g}}+\alpha_{\ell} \rho_{\ell} v_{\ell}\right)=\mathrm{d} u_{2}+\mathrm{d} u_{3} .
$$

Then for the second component

$$
\mathrm{d} f_{2}=\mathrm{d}\left(\alpha_{\mathrm{g}} \rho_{\mathrm{g}} v_{\mathrm{g}}^{2}\right)=v_{\mathrm{g}} \mathrm{d}\left(\alpha_{\mathrm{g}} \rho_{\mathrm{g}} v_{\mathrm{g}}\right)+\alpha_{\mathrm{g}} \rho_{\mathrm{g}} v_{\mathrm{g}} \mathrm{d} v_{\mathrm{g}}=v_{\mathrm{g}} \mathrm{d} u_{2}+\alpha_{\mathrm{g}} \rho_{\mathrm{g}} v_{\mathrm{g}} \mathrm{d} v_{\mathrm{g}},
$$

where $\mathrm{d} v_{\mathrm{g}}$ is substituted using (4.31)

$$
\begin{aligned}
\mathrm{d} f_{2}= & -\frac{\rho_{\mathrm{g}} v_{\mathrm{g}}^{2}}{\rho_{\mathrm{g}}-\rho_{\ell}} \mathrm{d} u_{1}+2 v_{\mathrm{g}} \mathrm{d} u_{2}+\frac{1}{\Phi} \frac{v_{\mathrm{g}}^{2}}{\rho_{\mathrm{g}}-\rho_{\ell}} \\
& \times\left(\alpha_{\mathrm{g}} \rho_{\ell} \frac{\Psi_{\mathrm{g}}}{c_{\mathrm{g}}^{2}}+\alpha_{\ell} \rho_{\mathrm{g}} \frac{\Psi_{\ell}}{c_{\ell}^{2}}\right)\left(\frac{\mathcal{E}}{\rho_{\mathrm{g}}-\rho_{\ell}} \mathrm{d} u_{1}-v_{\mathrm{g}} \mathrm{d} u_{2}-v_{\ell} \mathrm{d} u_{3}+\mathrm{d} u_{4}\right) .
\end{aligned}
$$

Similarly, for the third component

$$
\mathrm{d} f_{3}=v_{\ell} \mathrm{d} u_{3}+\alpha_{\ell} \rho_{\ell} v_{\ell} \mathrm{d} v_{\ell}
$$

where $\mathrm{d} v_{\ell}$ is substituted using (4.32)

$$
\begin{aligned}
\mathrm{d} f_{3}= & \frac{\rho_{\ell} v_{\ell}^{2}}{\rho_{\mathrm{g}}-\rho_{\ell}} \mathrm{d} u_{1}+2 v_{\ell} \mathrm{d} u_{3}-\frac{1}{\Phi} \frac{v_{\ell}^{2}}{\rho_{\mathrm{g}}-\rho_{\ell}} \\
& \times\left(\alpha_{\mathrm{g}} \rho_{\ell} \frac{\Psi_{\mathrm{g}}}{c_{\mathrm{g}}^{2}}+\alpha_{\ell} \rho_{\mathrm{g}} \frac{\Psi_{\ell}}{c_{\ell}^{2}}\right)\left(\frac{\mathcal{E}}{\rho_{\mathrm{g}}-\rho_{\ell}} \mathrm{d} u_{1}-v_{\mathrm{g}} \mathrm{d} u_{2}-v_{\ell} \mathrm{d} u_{3}+\mathrm{d} u_{4}\right)
\end{aligned}
$$

Finally, the fourth component can be written as

$$
\begin{aligned}
\mathrm{d} f_{4}=\frac{1}{2} v_{\mathrm{g}}^{2} \mathrm{~d} u_{2}+\frac{1}{2} v_{\ell}^{2} \mathrm{~d} u_{3}+\left(\rho_{\mathrm{g}} e_{\mathrm{g}} v_{\mathrm{g}}+v_{\mathrm{g}} p-\rho_{\ell} e_{\ell} v_{\ell}-v_{\ell} p\right) \mathrm{d} \alpha_{\mathrm{g}} \\
+\left(\alpha_{\mathrm{g}} v_{\mathrm{g}}+\alpha_{\ell} v_{\ell}\right) \mathrm{d} p+\alpha_{\mathrm{g}} e_{\mathrm{g}} v_{\mathrm{g}} \mathrm{d} \rho_{\mathrm{g}}+\alpha_{\ell} e_{\ell} v_{\ell} \mathrm{d} \rho_{\ell}+\alpha_{\mathrm{g}} \rho_{\mathrm{g}} v_{\mathrm{g}} \mathrm{d} e_{\mathrm{g}}+\alpha_{\ell} \rho_{\ell} v_{\ell} \mathrm{d} e_{\ell} \\
+\left(\alpha_{\mathrm{g}} \rho_{\mathrm{g}}\left(e_{\mathrm{g}}+v_{\mathrm{g}}^{2}\right)+\alpha_{\mathrm{g}} p\right) \mathrm{d} v_{\mathrm{g}}+\left(\alpha_{\ell} \rho_{\ell}\left(e_{\ell}+v_{\ell}^{2}\right)+\alpha_{\ell} p\right) \mathrm{d} v_{\ell},
\end{aligned}
$$


which after replacement of the differentials and simplification becomes

$$
\begin{aligned}
\mathrm{d} f_{4}= & \frac{-\rho_{\mathrm{g}} v_{\mathrm{g}}^{3}+\rho_{\ell} v_{\ell}^{3}}{\rho_{\mathrm{g}}-\rho_{\ell}} \mathrm{d} u_{1}+\left(e_{\mathrm{g}}+\frac{3}{2} v_{\mathrm{g}}^{2}+\frac{p}{\rho_{\mathrm{g}}}\right) \mathrm{d} u_{2}+\left(e_{\ell}+\frac{3}{2} v_{\ell}^{2}+\frac{p}{\rho_{\ell}}\right) \mathrm{d} u_{3} \\
& +\frac{1}{\Phi}\left[\frac{v_{\mathrm{g}}^{3}-v_{\ell}^{3}}{\rho_{\mathrm{g}}-\rho_{\ell}}\left(\alpha_{\mathrm{g}} \rho_{\ell} \frac{\Psi_{\mathrm{g}}}{c_{\mathrm{g}}^{2}}+\alpha_{\ell} \rho_{\mathrm{g}} \frac{\Psi_{\ell}}{c_{\ell}^{2}}\right)+\alpha_{\mathrm{g}} v_{\mathrm{g}}+\alpha_{\ell} v_{\ell}-T\left(\alpha_{\mathrm{g}} \rho_{\mathrm{g}} v_{\mathrm{g}} C_{p, \mathrm{~g}} \chi_{\mathrm{g}}+\alpha_{\ell} \rho_{\ell} v_{\ell} C_{p, \ell} \chi_{\ell}\right)\right] \\
& \times\left(\frac{\mathcal{E}}{\rho_{\mathrm{g}}-\rho_{\ell}} \mathrm{d} u_{1}-v_{\mathrm{g}} \mathrm{d} u_{2}-v_{\ell} \mathrm{d} u_{3}+\mathrm{d} u_{4}\right) .
\end{aligned}
$$

Similarly, for the non-conservative part of the fluxes, we need to derive a Jacobian matrix for the vector $\boldsymbol{W}$. First, we can remark that

$$
\mathrm{d} w_{1}=\mathrm{d} p
$$

which gives after substitution of the differentials

$$
\mathrm{d} w_{1}=\frac{1}{\Phi}\left(\frac{\mathcal{E}}{\rho_{\mathrm{g}}-\rho_{\ell}} \mathrm{d} u_{1}-v_{\mathrm{g}} \mathrm{d} u_{2}-v_{\ell} \mathrm{d} u_{3}+\mathrm{d} u_{4}\right) .
$$

For the second component, we have that

$$
\mathrm{d} w_{2}=\mathrm{d}\left(\alpha_{\mathrm{g}} v_{\mathrm{g}}+\alpha_{\ell} v_{\ell}\right)=\frac{1}{\rho_{\mathrm{g}}}\left(\mathrm{d} u_{2}-\alpha_{\mathrm{g}} v_{\mathrm{g}} \mathrm{d} \rho_{\mathrm{g}}\right)+\frac{1}{\rho_{\ell}}\left(\mathrm{d} u_{3}-\alpha_{\ell} v_{\ell} \mathrm{d} \rho_{\ell}\right),
$$

which gives

$$
\mathrm{d} w_{2}=\frac{1}{\rho_{\mathrm{g}}} \mathrm{d} u_{2}+\frac{1}{\rho_{\ell}} \mathrm{d} u_{3}-\frac{1}{\Phi}\left(\alpha_{\mathrm{g}} v_{\mathrm{g}} \frac{\Psi_{\mathrm{g}}}{\rho_{\mathrm{g}} c_{\mathrm{g}}^{2}}+\alpha_{\ell} v_{\ell} \frac{\Psi_{\ell}}{\rho_{\ell} c_{\ell}^{2}}\right)\left(\frac{\mathcal{E}}{\rho_{\mathrm{g}}-\rho_{\ell}} \mathrm{d} u_{1}-v_{\mathrm{g}} \mathrm{d} u_{2}-v_{\ell} \mathrm{d} u_{3}+\mathrm{d} u_{4}\right) .
$$

Finally, the third component is the volume fraction differential (4.26)

$$
\mathrm{d} w_{3}=\mathrm{d} \alpha_{\mathrm{g}},
$$

thus

$$
\mathrm{d} w_{3}=\frac{1}{\rho_{\mathrm{g}}-\rho_{\ell}} \mathrm{d} u_{1}-\frac{1}{\rho_{\mathrm{g}}-\rho_{\ell}} \frac{1}{\Phi}\left(\alpha_{\mathrm{g}} \frac{\Psi_{\mathrm{g}}}{c_{\mathrm{g}}^{2}}+\alpha_{\ell} \frac{\Psi_{\ell}}{c_{\ell}^{2}}\right)\left(\frac{\mathcal{E}}{\rho_{\mathrm{g}}-\rho_{\ell}} \mathrm{d} u_{1}-v_{\mathrm{g}} \mathrm{d} u_{2}-v_{\ell} \mathrm{d} u_{3}+\mathrm{d} u_{4}\right) .
$$

\subsection{The matrices in the quasilinear form}

We can now write the matrix $\boldsymbol{A}(\boldsymbol{U})$ appearing in the quasilinear form (4.1). Following a flux-splitting strategy (see for example [13]), we may split the matrix in a conservative part and a non-conservative part. With the help of (4.33), (4.35), (4.37) and (4.39), the conservative part is written as

$$
\boldsymbol{A}_{c}(\boldsymbol{U})=\frac{\partial \boldsymbol{F}_{c}(\boldsymbol{U})}{\partial \boldsymbol{U}}=\left(\begin{array}{cccc}
0 & 1 & 1 & 0 \\
-\frac{\rho_{\mathrm{g}} v_{\mathrm{g}}^{2}}{\rho_{\mathrm{g}}-\rho_{\ell}}+\frac{v_{\mathrm{g}}^{2} \mathcal{E}}{\rho_{\mathrm{g}}-\rho_{\ell}} \Sigma_{\rho} & 2 v_{\mathrm{g}}-v_{\mathrm{g}}^{3} \Sigma_{\rho} & -v_{\mathrm{g}}^{2} v_{\ell} \Sigma_{\rho} & v_{\mathrm{g}}^{2} \Sigma_{\rho} \\
\frac{\rho_{\ell} v_{\ell}^{2}}{\rho_{\mathrm{g}}-\rho_{\ell}}-\frac{v_{\ell}^{2} \mathcal{E}}{\rho_{\mathrm{g}}-\rho_{\ell}} \Sigma_{\rho} & v_{\mathrm{g}} v_{\ell}^{2} \Sigma_{\rho} & 2 v_{\ell}+v_{\ell}^{3} \Sigma_{\rho} & -v_{\ell}^{2} \Sigma_{\rho} \\
a_{41} & a_{42} & a_{43} & \left(v_{\mathrm{g}}^{3}-v_{\ell}^{3}\right) \Sigma_{\rho}+\Omega
\end{array}\right)
$$


where

$$
\begin{aligned}
a_{41} & =\frac{-\rho_{\mathrm{g}} v_{\mathrm{g}}^{3}+\rho_{\ell} v_{\ell}^{3}}{\rho_{\mathrm{g}}-\rho_{\ell}}+\left(\left(v_{\mathrm{g}}^{3}-v_{\ell}^{3}\right) \Sigma_{\rho}+\Omega\right) \frac{\mathcal{E}}{\rho_{\mathrm{g}}-\rho_{\ell}}, \\
a_{42} & =\left(e_{\mathrm{g}}+\frac{3}{2} v_{\mathrm{g}}^{2}+\frac{p}{\rho_{\mathrm{g}}}\right)-\left(\left(v_{\mathrm{g}}^{3}-v_{\ell}^{3}\right) \Sigma_{\rho}+\Omega\right) v_{\mathrm{g}}, \\
a_{43} & =\left(e_{\ell}+\frac{3}{2} v_{\ell}^{2}+\frac{p}{\rho_{\ell}}\right)-\left(\left(v_{\mathrm{g}}^{3}-v_{\ell}^{3}\right) \Sigma_{\rho}+\Omega\right) v_{\ell} .
\end{aligned}
$$

We have also introduced the shorthands

$$
\Sigma_{\rho}=\frac{1}{\Phi} \frac{1}{\left(\rho_{\mathrm{g}}-\rho_{\ell}\right)}\left(\alpha_{\mathrm{g}} \rho_{\ell} \frac{\Psi_{\mathrm{g}}}{c_{\mathrm{g}}^{2}}+\alpha_{\ell} \rho_{\mathrm{g}} \frac{\Psi_{\ell}}{c_{\ell}^{2}}\right)
$$

and

$$
\Omega=\frac{1}{\Phi}\left(\alpha_{\mathrm{g}} v_{\mathrm{g}}+\alpha_{\ell} v_{\ell}-\alpha_{\mathrm{g}} \rho_{\mathrm{g}} v_{\mathrm{g}} T C_{p, \mathrm{~g}} \chi_{\mathrm{g}}-\alpha_{\ell} \rho_{\ell} v_{\ell} T C_{p, \ell} \chi_{\ell}\right) .
$$

For the non-conservative part, we can express the Jacobian of the vector $\boldsymbol{W}(\boldsymbol{U})$ using (4.41), (4.43) and (4.45)

$$
\boldsymbol{M}(\boldsymbol{U})=\frac{\partial \boldsymbol{W}(\boldsymbol{U})}{\partial \boldsymbol{U}}=\left(\begin{array}{cccc}
\frac{1}{\Phi} \frac{\mathcal{E}}{\rho_{\mathrm{g}}-\rho_{\ell}} & -\frac{v_{\mathrm{g}}}{\Phi} & -\frac{v_{\ell}}{\Phi} & \frac{1}{\Phi} \\
-\frac{\mathcal{E}}{\rho_{\mathrm{g}}-\rho_{\ell}} \Sigma_{v} & \frac{1}{\rho_{\mathrm{g}}}+v_{\mathrm{g}} \Sigma_{v} & \frac{1}{\rho_{\ell}}+v_{\ell} \Sigma_{v} & -\Sigma_{v} \\
\frac{1}{\rho_{\mathrm{g}}-\rho_{\ell}}-\frac{\mathcal{E}}{\rho_{\mathrm{g}}-\rho_{\ell}} \Sigma & v_{\mathrm{g}} \Sigma & v_{\ell} \Sigma & -\Sigma
\end{array}\right)
$$

where

$$
\begin{aligned}
\Sigma & =\frac{1}{\Phi} \frac{1}{\rho_{\mathrm{g}}-\rho_{\ell}}\left(\alpha_{\mathrm{g}} \frac{\Psi_{\mathrm{g}}}{c_{\mathrm{g}}^{2}}+\alpha_{\ell} \frac{\Psi_{\ell}}{c_{\ell}^{2}}\right), \\
\Sigma_{v} & =\frac{1}{\Phi}\left(\alpha_{\mathrm{g}} v_{\mathrm{g}} \frac{\Psi_{\mathrm{g}}}{\rho_{\mathrm{g}} c_{\mathrm{g}}^{2}}+\alpha_{\ell} v_{\ell} \frac{\Psi_{\ell}}{\rho_{\ell} c_{\ell}^{2}}\right) .
\end{aligned}
$$

The Jacobian of the non-conservative fluxes then follows from

$$
\boldsymbol{A}_{p}(\boldsymbol{U})=\boldsymbol{B}(\boldsymbol{U}) \cdot \boldsymbol{M}(\boldsymbol{U}) .
$$

The Jacobian of the whole system is then

$$
\boldsymbol{A}(\boldsymbol{U})=\boldsymbol{A}_{c}(\boldsymbol{U})+\boldsymbol{A}_{p}(\boldsymbol{U}) .
$$

\section{SUbChARACTERISTIC CONDITION}

The stiff limit of a relaxation model is linearly stable if and only if the equilibrium eigenvalues interlace those of the relaxation system $[9,42]$. This criterion is known as the subcharacteristic condition, and is implied by the stronger stability criteria given in $[7,9]$. In particular, stability requires that the wave speeds of the equilibrium system do not exceed the maximum wave speed of the relaxation system $[9,15,26,28,34]$.

We expect the two-fluid models mentioned in the present paper to respect this condition when the underlying physical models describe a stable reality. Figure 1 presents the model hierarchy, where $\mathrm{TF}_{n}$ and $\mathrm{DF}_{n}$, respectively, denote the two-fluid and the drift-flux models, and the index $n$ the number of conservation equations in the model. The complete hierarchy can be found in [25]. Each arrow designates the relaxation performed from one model to the next. Specifically for the model (2.1)-(2.5), the $\mu$-relaxation means to let $\mathcal{K} \rightarrow \infty$, while the $v$-relaxation means to let $\mathcal{F} \rightarrow \infty$. The subcharacteristic condition has been proved for some of the relaxation 


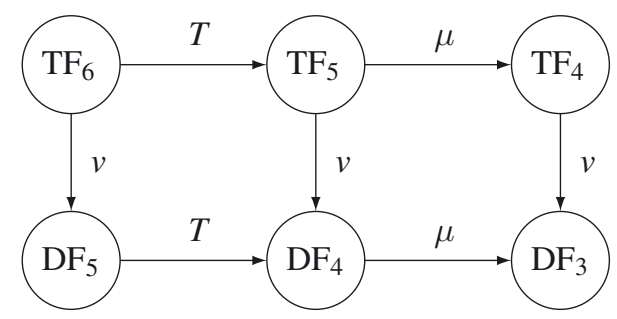

Figure 1. A hierarchy of two-phase flow models. TF: Two-phase model. DF: Drift-flux model. Index: Number of conservation equations.

processes by [29] and [15]. In the present section, we prove a subcharacteristic condition for the remaining relaxation processes $\mathrm{TF}_{5} \rightarrow \mathrm{TF}_{4}$ and $\mathrm{TF}_{4} \rightarrow \mathrm{DF}_{3}$.

Herein, the notation $\mathrm{TF}_{4}$ refers to the model described in this paper (Sect. 3.4). The model $\mathrm{TF}_{5}$ is considered in [29], and constitutes our starting point (2.1)-(2.5). The model $\mathrm{DF}_{3}$ is the full equilibrium model considered for instance in Section 6 of [15]. We refer to [15,29] for a more detailed description of the remaining models in the hierarchy.

\subsection{Speed of sound}

The eigenvalues of the Jacobian of the fluxes $\boldsymbol{A}(\boldsymbol{U})$ are the propagation velocities of the quantities defined by the eigenvectors of $\boldsymbol{A}(\boldsymbol{U})$, also called waves. In the present model, these waves are the volume-fraction waves and the pressure waves.

For the purposes of the current paper, we define the two-phase speed of sound as the speed of pressure waves relative to a reference frame moving with the average velocity of the waves. That is, for a two-phase mixture we define $c$ and $v_{\mathrm{m}}$ through

$$
\begin{aligned}
& \lambda^{p+}=v_{\mathrm{m}}+c, \\
& \lambda^{p-}=v_{\mathrm{m}}-c
\end{aligned}
$$

where $\lambda^{p+}$ is the velocity of the right-going pressure wave and $\lambda^{p-}$ is the velocity of the left-going pressure wave. For subsonic flows in the models we consider, the pressure waves will be the fastest waves. Hence $v_{\mathrm{m}}$ and $c$ are uniquely determined through

$$
\begin{aligned}
v_{\mathrm{m}} & =\frac{1}{2}\left(\lambda_{\max }+\lambda_{\min }\right), \\
c & =\frac{1}{2}\left(\lambda_{\max }-\lambda_{\min }\right) .
\end{aligned}
$$

Note that this is a fluid-mechanical definition of speed of sound, which does not necessarily coincide with the thermodynamical expression

$$
c^{2}=\left(\frac{\partial p}{\partial \rho}\right)_{s}
$$

for mixture variables $\rho$ and $s$. In fact, for two-fluid models the definition (5.4) of $c$ will generally imply that $c$ depends on the relative velocity $\left(v_{\mathrm{g}}-v_{\ell}\right)$.

For $v_{\mathrm{g}} \neq v_{\ell}$, it is well-known [44] that no tractable exact expression exists for the eigenvalues. Hence our following eigenvalue analysis will be limited to $v_{\mathrm{g}}=v_{\ell}$. 
Proposition 5.1. When the liquid and gas velocities are equal to each other, the eigenvalues of the two-fluid four-equation model are

$$
\boldsymbol{\Lambda}_{T F 4}=\left(\begin{array}{c}
v_{\mathrm{m}}-c_{T F 4} \\
v_{\mathrm{m}} \\
v_{\mathrm{m}} \\
v_{\mathrm{m}}+c_{T F_{4}}
\end{array}\right),
$$

where the velocities have been substituted by $v_{\mathrm{g}}=v_{\mathrm{m}}$ and $v_{\ell}=v_{\mathrm{m}}$, and the speed of sound of the model is given by

$$
c_{T F 4}=\sqrt{\frac{\alpha_{\ell} \rho_{\mathrm{g}}+\alpha_{\mathrm{g}} \rho_{\ell}}{\rho_{\mathrm{g}} \rho_{\ell}\left(\frac{\alpha_{\mathrm{g}}}{\rho_{\mathrm{g}} c_{\mathrm{g}}^{2}}+\frac{\alpha_{\ell}}{\rho_{\ell} c_{\ell}^{2}}+T\left(\alpha_{\mathrm{g}} \rho_{\mathrm{g}} C_{p, \mathrm{~g}} \chi_{\mathrm{g}}^{2}+\alpha_{\ell} \rho_{\ell} C_{p, \ell} \chi_{\ell}^{2}\right)\right)}} .
$$

Proof. When $v_{\mathrm{g}}=0$ and $v_{\ell}=0$, the matrix $\boldsymbol{A}(\boldsymbol{U})$ becomes

$$
\boldsymbol{A}\left(\boldsymbol{U}\left(v_{\mathrm{g}}=0, v_{\ell}=0\right)\right)=\left(\begin{array}{cccc}
0 & 1 & 1 & 0 \\
\frac{\alpha_{\mathrm{g}}\left(\rho_{\mathrm{g}} e_{\mathrm{g}}-\rho_{\ell} e_{\ell}\right) c_{\mathrm{TF} 4}^{2}}{\left(\alpha_{\ell} \rho_{\mathrm{g}}+\alpha_{\mathrm{g}} \rho_{\ell}\right) T\left(s_{\mathrm{g}}-s_{\ell}\right)} & 0 & 0 & -\frac{\alpha_{\mathrm{g}}\left(\rho_{\mathrm{g}}-\rho_{\ell}\right) c_{\mathrm{TF} 4}^{2}}{\left(\alpha_{\ell} \rho_{\mathrm{g}}+\alpha_{\mathrm{g}} \rho_{\ell}\right) T\left(s_{\mathrm{g}}-s_{\ell}\right)} \\
\frac{\alpha_{\ell}\left(\rho_{\mathrm{g}} e_{\mathrm{g}}-\rho_{\ell} e_{\ell}\right) c_{\mathrm{TF} 4}^{2}}{\left(\alpha_{\ell} \rho_{\mathrm{g}}+\alpha_{\mathrm{g}} \rho_{\ell}\right) T\left(s_{\mathrm{g}}-s_{\ell}\right)} & 0 & 0 & -\frac{\alpha_{\ell}\left(\rho_{\mathrm{g}}-\rho_{\ell}\right) c_{\mathrm{TF} 4}^{2}}{\left(\alpha_{\ell} \rho_{\mathrm{g}}+\alpha_{\mathrm{g}} \rho_{\ell}\right) T\left(s_{\mathrm{g}}-s_{\ell}\right)} \\
0 & e_{\mathrm{g}}+\frac{p}{\rho_{\mathrm{g}}} & e_{\ell}+\frac{p}{\rho_{\ell}} & 0
\end{array}\right),
$$

where

$$
c_{\mathrm{TF} 4}=\sqrt{\frac{\alpha_{\ell} \rho_{\mathrm{g}}+\alpha_{\mathrm{g}} \rho_{\ell}}{\rho_{\mathrm{g}} \rho_{\ell}\left(\frac{\alpha_{\mathrm{g}}}{\rho_{\mathrm{g}} c_{\mathrm{g}}^{2}} \Psi_{\mathrm{g}}+\frac{\alpha_{\ell}}{\rho_{\ell} c_{\ell}^{2}} \Psi_{\ell}+T\left(\alpha_{\mathrm{g}} \rho_{\mathrm{g}} C_{p, \mathrm{~g}} \chi_{\mathrm{g}}+\alpha_{\ell} \rho_{\ell} C_{p, \ell} \chi_{\ell}\right) \frac{\rho_{\mathrm{g}}-\rho_{\ell}}{\rho_{\mathrm{g}} \rho_{\ell} L}\right)}} .
$$

Its eigenvalues are then $0,0, c_{\mathrm{TF} 4}$ and $-c_{\mathrm{TF} 4}$. The waves with zero velocity are the volume-fraction waves, while the two other are the pressure waves. We deduce that $c_{\mathrm{TF} 4}$ is the speed of sound of the model. This speed of sound is dependent on the thermodynamical assumptions, here that the phases are at all times at equilibrium. The expression (5.9) uses the variable blocks that are involved in the Jacobian matrices. We can also reorganise it to the more compact form 5.7.

Note that the speed of sound can be used to simplify (3.25) and (3.26)

$$
\begin{aligned}
\mathcal{V} & =\frac{\rho_{\mathrm{g}} \rho_{\ell}}{\alpha_{\ell} \rho_{\mathrm{g}}+\alpha_{\mathrm{g}} \rho_{\ell}} \frac{T}{L}\left(\alpha_{\mathrm{g}} \rho_{\mathrm{g}} C_{p, \mathrm{~g}} \chi_{\mathrm{g}}+\alpha_{\ell} \rho_{\ell} C_{p, \ell} \chi_{\ell}\right) c_{\mathrm{TF} 4}^{2}, \\
\mathcal{P} & =\frac{\alpha_{\mathrm{g}} \alpha_{\ell} \rho_{\mathrm{g}} \rho_{\ell}\left(v_{\mathrm{g}}-v_{\ell}\right)}{\alpha_{\ell} \rho_{\mathrm{g}}+\alpha_{\mathrm{g}} \rho_{\ell}} \frac{T}{L}\left(\rho_{\ell} C_{p, \ell} \chi_{\ell} \frac{\Psi_{\mathrm{g}}}{\rho_{\mathrm{g}} c_{\mathrm{g}}^{2}}-\rho_{\mathrm{g}} C_{p, \mathrm{~g}} \chi_{\mathrm{g}} \frac{\Psi_{\ell}}{\rho_{\ell} c_{\ell}^{2}}\right) c_{\mathrm{TF} 4}^{2} .
\end{aligned}
$$

The eigenstructure for the general case is not accessible. However, when $v_{\mathrm{g}}=v_{\ell}$, we are able to find the exact eigenvalues of the system. For this, we write the characteristic polynomial of the matrix $\boldsymbol{A}(\boldsymbol{U})$ where the velocities have been substituted with $v_{\mathrm{g}}=v_{\mathrm{m}}$ and $v_{\ell}=v_{\mathrm{m}}$

$$
\Pi_{A, v_{\mathrm{g}}=v_{\ell}}=\operatorname{Det}\left(\boldsymbol{A}\left(\boldsymbol{U}_{v_{\mathrm{g}}=v_{\ell}}\right)-\lambda \cdot \boldsymbol{I}_{4}\right),
$$

where $\boldsymbol{I}_{4}$ is the identity matrix of rank 4 . This polynomial can be simplified to

$$
\Pi_{A, v_{\mathrm{g}}=v_{\ell}}=\left(\lambda-v_{\mathrm{m}}\right)^{2} \cdot\left(\lambda-\left(v_{\mathrm{m}}+c_{\mathrm{TF} 4}\right)\right) \cdot\left(\lambda-\left(v_{\mathrm{m}}-c_{\mathrm{TF} 4}\right)\right),
$$

which is solved by the eigenvalues presented in (5.6). 


\subsection{Speed of sound in other models}

The speed of sound of the five-equation model is given by [29]. In order to express it in terms of the parameters used in the present article, we first derive a relation. In [29], the parameter

$$
\zeta=\left(\frac{\partial T}{\partial p}\right)_{s}=-\frac{1}{\rho^{2}}\left(\frac{\partial \rho}{\partial s}\right)_{p}
$$

is used. The triple product rule gives

$$
\zeta=\frac{1}{\rho^{2}}\left(\frac{\partial p}{\partial s}\right)_{\rho} /\left(\frac{\partial p}{\partial \rho}\right)_{s}
$$

where

$$
\left(\frac{\partial p}{\partial \rho}\right)_{s}=c^{2}
$$

and, from [32],

$$
\left(\frac{\partial p}{\partial s}\right)_{\rho}=\left(\frac{\partial p}{\partial e}\right)_{\rho}\left(\frac{\partial e}{\partial s}\right)_{\rho} \stackrel{\Gamma \equiv \frac{1}{\rho}\left(\frac{\partial p}{\partial e}\right)_{\rho}}{=} \rho \Gamma\left(\frac{\partial e}{\partial s}\right)_{\rho} \stackrel{d e=T d s-p / \rho^{2} d \rho}{=} \Gamma \rho T
$$

Thus

$$
\zeta=\frac{\Gamma T}{\rho c^{2}}
$$

The speed of sound in the five-equation model, taken from [29] and simplified, is

$$
c_{\mathrm{TF} 5}=\sqrt{\frac{\alpha_{\mathrm{g}} \rho_{\ell}+\alpha_{\ell} \rho_{\mathrm{g}}}{\rho_{\mathrm{g}} \rho_{\ell}\left(\frac{\alpha_{\mathrm{g}}}{\rho_{\mathrm{g}} c_{\mathrm{g}}^{2}}+\frac{\alpha_{\ell}}{\rho_{\ell} c_{\ell}^{2}}+\frac{\alpha_{\mathrm{g}} \rho_{\mathrm{g}} C_{p, \mathrm{~g}} \alpha_{\ell} \rho_{\ell} C_{p, \ell} T\left(\frac{\Gamma_{\mathrm{g}}}{\rho_{\mathrm{g}} c_{\mathrm{g}}^{2}}-\frac{\Gamma_{\ell}}{\rho_{\ell} c_{\ell}^{2}}\right)^{2}}{\alpha_{\mathrm{g}} \rho_{\mathrm{g}} C_{p, \mathrm{~g}}+\alpha_{\ell} \rho_{\ell} C_{p, \ell}}\right)}} .
$$

We also know from [15] the speed of sound in the drift-flux three-equation model. This model can be seen as the limit of the drift-flux four-equation model with instantaneous phase relaxation, or as the limit of the two-fluid four-equation model (3.34)-(3.37) with instantaneous velocity relaxation. This is obtained by summing equations (3.35) and (3.36) and assuming $v_{\mathrm{g}}=v_{\ell}$. After simplification, the speed of sound can be written

$$
c_{\mathrm{DF} 3}=\frac{1}{\sqrt{\left(\alpha_{\mathrm{g}} \rho_{\mathrm{g}}+\alpha_{\ell} \rho_{\ell}\right)\left(\frac{\alpha_{\mathrm{g}}}{\rho_{\mathrm{g}} c_{\mathrm{g}}^{2}}+\frac{\alpha_{\ell}}{\rho_{\ell} c_{\ell}^{2}}+T\left(\alpha_{\mathrm{g}} \rho_{\mathrm{g}} C_{p, \mathrm{~g}} \chi_{\mathrm{g}}^{2}+\alpha_{\ell} \rho_{\ell} C_{p, \ell} \chi_{\ell}^{2}\right)\right)}} .
$$

Proof. According to [15], the speed of sound in the drift-flux three-equation model is

$$
c_{\mathrm{DF} 3}^{-2}=c_{\mathrm{DF} 4}^{-2}+\left[\frac{\left(\alpha_{\mathrm{g}} \rho_{\mathrm{g}}+\alpha_{\ell} \rho_{\ell}\right) T}{\alpha_{\mathrm{g}} \rho_{\mathrm{g}} C_{p, \mathrm{~g}}+\alpha_{\ell} \rho_{\ell} C_{p, \ell}}\left(\frac{\rho_{\mathrm{g}}-\rho_{\ell}}{\rho_{\mathrm{g}} \rho_{\ell}\left(h_{\mathrm{g}}-h_{\ell}\right)}\left(\alpha_{\mathrm{g}} \rho_{\mathrm{g}} C_{p, \mathrm{~g}}+\alpha_{\ell} \rho_{\ell} C_{p, \ell}\right)+\frac{\Gamma_{\mathrm{g}} \alpha_{\mathrm{g}} \rho_{\mathrm{g}} C_{p, \mathrm{~g}}}{\rho_{\mathrm{g}} c_{\mathrm{g}}^{2}}+\frac{\Gamma_{\ell} \alpha_{\ell} \rho_{\ell} C_{p, \ell}}{\rho_{\ell} c_{\ell}^{2}}\right)^{2}\right],
$$

where $c_{\mathrm{DF} 4}$ is the speed of sound in the drift-flux model with instantaneous pressure and temperature relaxation. Substituting the definition of $\chi_{k}$ (Eq. (3.16)) in $c_{\mathrm{DF} 3}$, as well as $h_{\mathrm{g}}-h_{\ell}=L$, gives

$$
c_{\mathrm{DF} 3}^{-2}=c_{\mathrm{DF} 4}^{-2}+\frac{\left(\alpha_{\mathrm{g}} \rho_{\mathrm{g}}+\alpha_{\ell} \rho_{\ell}\right) T}{\alpha_{\mathrm{g}} \rho_{\mathrm{g}} C_{p, \mathrm{~g}}+\alpha_{\ell} \rho_{\ell} C_{p, \ell}}\left(\alpha_{\mathrm{g}} \rho_{\mathrm{g}} C_{p, \mathrm{~g}} \chi_{\mathrm{g}}+\alpha_{\ell} \rho_{\ell} C_{p, \ell} \chi_{\ell}\right)^{2} .
$$


Now, the speed of sound $c_{\mathrm{DF} 4}$ is $[15]$

$$
c_{\mathrm{DF} 4}^{-2}=c_{\mathrm{DF} 5}^{-2}+\rho T \frac{\alpha_{\mathrm{g}} \rho_{\mathrm{g}} C_{p, \mathrm{~g}} \alpha_{\ell} \rho_{\ell} C_{p, \ell}}{\alpha_{\mathrm{g}} \rho_{\mathrm{g}} C_{p, \mathrm{~g}}+\alpha_{\ell} \rho_{\ell} C_{p, \ell}}\left(\frac{\Gamma_{\ell}}{\rho_{\ell} c_{\ell}^{2}}-\frac{\Gamma_{\mathrm{g}}}{\rho_{\mathrm{g}} c_{\mathrm{g}}^{2}}\right)^{2},
$$

where $c_{\mathrm{DF} 5}$ is the speed of sound in the drift-flux model with instantaneous pressure equilibrium. Substituting the definition of $\chi_{k}$ (Eq. (3.16)) in $c_{\mathrm{DF} 4}$, as well as $c_{\mathrm{DF} 5}$ from ([15], Eq. (3.9)), gives

$$
c_{\mathrm{DF} 4}^{-2}=\rho\left(\frac{\alpha_{\mathrm{g}}}{\rho_{\mathrm{g}} c_{\mathrm{g}}^{2}}+\frac{\alpha_{\ell}}{\rho_{\ell} c_{\ell}^{2}}\right)+\rho T \frac{\alpha_{\mathrm{g}} \rho_{\mathrm{g}} C_{p, \mathrm{~g}} \alpha_{\ell} \rho_{\ell} C_{p, \ell}}{\alpha_{\mathrm{g}} \rho_{\mathrm{g}} C_{p, \mathrm{~g}}+\alpha_{\ell} \rho_{\ell} C_{p, \ell}}\left(\chi_{\ell}-\chi_{\mathrm{g}}\right)^{2} .
$$

Substituting this result in equation (5.22) and simplifying gives the result.

\subsection{Comparison of the speeds of sound}

Ferrer et al. [29] compared the speeds of sound of four of the two-phase flow models in Figure $1-$ the $\mathrm{TF}_{6}$, $\mathrm{TF}_{5}, \mathrm{DF}_{5}$ and $\mathrm{DF}_{4}$ models. They showed that the effect of the instantaneous relaxation of a given type on the mixture speed of sound is independent of the order in which relaxations are performed. For example, the effect of relaxing the velocity multiplies the speed of sound by a constant factor

$$
\frac{c_{\mathrm{TF} 5}}{c_{\mathrm{DF} 4}}=\frac{c_{\mathrm{TF} 6}}{c_{\mathrm{DF} 5}}=\sqrt{\left(\alpha_{\mathrm{g}} \rho_{\mathrm{g}}+\alpha_{\ell} \rho_{\ell}\right)\left(\frac{\alpha_{\mathrm{g}}}{\rho_{\mathrm{g}}}+\frac{\alpha_{\ell}}{\rho_{\ell}}\right)} .
$$

By rearranging the expression above, they also arrive at

$$
\frac{c_{\mathrm{DF} 5}}{c_{\mathrm{DF} 4}}=\frac{c_{\mathrm{TF} 6}}{c_{\mathrm{TF} 5}},
$$

which shows that the same conclusion applies to the effect of thermal relaxation.

Now, in the present work, we derived $\mathrm{TF}_{4}$ from the $\mathrm{TF}_{5}$ model previously mentioned by performing instantaneous phase relaxation, and found its sound speed (5.7). By comparing it to the speed of sound in the $\mathrm{DF}_{3}$ (5.20), we immediately see that we can extend the ratio relation (5.25) with

$$
\frac{c_{\mathrm{TF} 4}}{c_{\mathrm{DF} 3}}=\frac{c_{\mathrm{TF} 5}}{c_{\mathrm{DF} 4}}=\frac{c_{\mathrm{TF} 6}}{c_{\mathrm{DF} 5}},
$$

which shows that the velocity relaxation once more has an independent effect on the speed of sound. From the above relation, we can deduce

$$
\frac{c_{\mathrm{DF} 4}}{c_{\mathrm{DF} 3}}=\frac{c_{\mathrm{TF} 5}}{c_{\mathrm{TF} 4}},
$$

hence, the effect of phase relaxation on the sound speed is also independent from the order of the relaxation steps.

Using the results of [29] on the ordering of the speeds of sound, we can write from (5.27)

$$
c_{\mathrm{DF} 3} \leq c_{\mathrm{TF} 4} .
$$

Now, we take the difference between the two speeds of sound $c_{\mathrm{TF} 4}$ and $c_{\mathrm{TF} 5}$, or more precisely the inverse of their squares, which gives

$$
c_{\mathrm{TF} 4}^{-2}-c_{\mathrm{TF} 5}^{-2}=\frac{\rho_{\mathrm{g}} \rho_{\ell}}{\alpha_{\ell} \rho_{\mathrm{g}}+\alpha_{\mathrm{g}} \rho_{\ell}} \frac{T\left(\alpha_{\mathrm{g}} \rho_{\mathrm{g}} C_{p, \mathrm{~g}} \chi_{\mathrm{g}}+\alpha_{\ell} \rho_{\ell} C_{p, \ell} \chi_{\ell}\right)^{2}}{\alpha_{\mathrm{g}} \rho_{\mathrm{g}} C_{p, \mathrm{~g}}+\alpha_{\ell} \rho_{\ell} C_{p, \ell}} .
$$

This difference is always non-negative, which proves that

$$
c_{\mathrm{TF} 4} \leq c_{\mathrm{TF} 5} .
$$

Consequently, from (5.28)

$$
c_{\mathrm{DF} 3} \leq c_{\mathrm{DF} 4} .
$$


TABLE 2. Methane gas-liquid mixture under atmospheric pressure.

\begin{tabular}{lcc}
\hline Quantity & Gas & Liquid \\
\hline Temperature $(\mathrm{K})$ & 111.67 & 111.67 \\
Density $\left(\mathrm{kg} / \mathrm{m}^{3}\right)$ & 1.8164 & 422.36 \\
Sound speed $(\mathrm{m} / \mathrm{s})$ & 271.46 & 1338.1 \\
$C_{p}(\mathrm{~J} /(\mathrm{kg} \mathrm{K}))$ & 2217.7 & 3481.1 \\
Entropy $\left(\mathrm{m}^{2} /\left(\mathrm{s}^{2} \mathrm{~K}\right)\right)$ & 4574.6 & $-2.5 \times 10^{-10}$ \\
$\Gamma($ dimensionless $)$ & 0.333 & 1.78 \\
\hline
\end{tabular}

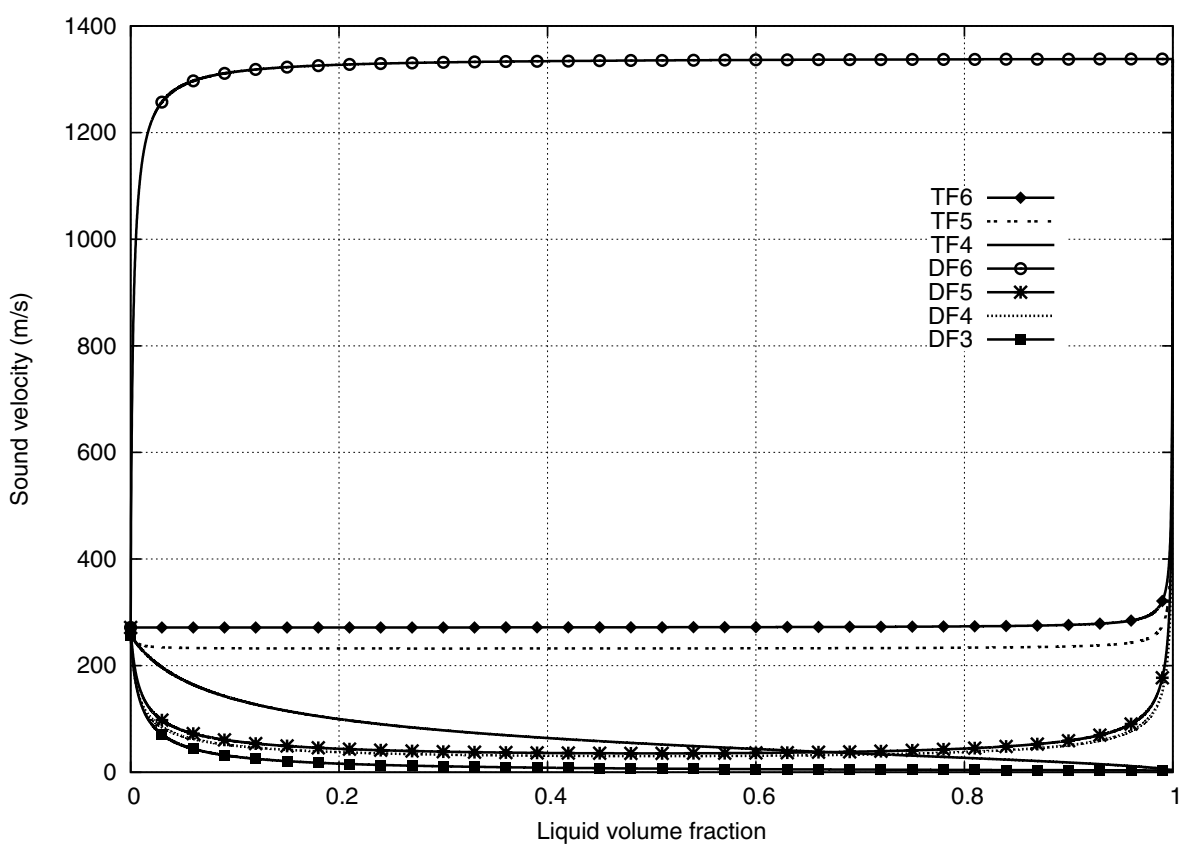

FiguRE 2. Gas-liquid methane mixture at atmospheric pressure. Mixture sound velocities for models with different equilibrium assumptions.

\subsubsection{A methane two-phase mixture}

In this section, we illustrate our results applied to a liquid-gas methane mixture at atmospheric pressure. Similar illustrations for $\mathrm{CO}_{2}$ and water-steam may be found in the references $[15,25,27,29]$.

As stated in Table 2, we apply physical parameters representative of methane at atmospheric pressure at the boiling point curve [41]. The various sound velocities are plotted in Figure 2 as functions of liquid volume fraction. Herein, DF6 refers to the basic 6-equation drift-flux model where only velocity equilibrium is imposed, i.e. the model has no relaxation in mass, volume or energy transfer [15].

We observe that imposing pressure equilibrium changes the mixture sound velocity to being dominated by gas rather than the liquid sound velocity. Temperature relaxation has little influence. Note also that the onephase limits of DF3 and TF4 are equal, but unlike the other models, do not correspond to the one-phase sound velocities. This discontinuity, particular to full thermodynamical relaxation, is well known and was commented also in $[5,15]$. 


\subsection{Subcharacteristic condition and model hierarchy}

Following the definition stated in [9] and the arguments of [15,29], we observe that for our models the subcharacteristic condition can be expressed as follows:

When the velocities in each phase are equal, model $X$ satisfies the subcharacteristic condition with respect to model $Y$ if and only if the mixture sound velocities satisfy

$$
c_{X} \leq c_{Y}
$$

Regarding the model hierarchy of Figure 1, we are now able to state the following results:

$\mathrm{SC1}$ : The model $\mathrm{DF}_{4}$ satisfies the subcharacteristic condition with respect to $\mathrm{DF}_{5}$.

$\mathrm{SC} 2$ : The model $\mathrm{DF}_{4}$ satisfies the subcharacteristic condition with respect to $\mathrm{TF}_{5}$.

SC3: The model $\mathrm{DF}_{5}$ satisfies the subcharacteristic condition with respect to $\mathrm{TF}_{6}$.

$\mathrm{SC} 4$ : When the velocities of each phase are equal, the model $\mathrm{TF}_{5}$ satisfies the subcharacteristic condition with respect to $\mathrm{TF}_{6}$.

SC5: The model $\mathrm{DF}_{3}$ satisfies the subcharacteristic condition with respect to $\mathrm{TF}_{4}$.

SC6: The model $\mathrm{DF}_{3}$ satisfies the subcharacteristic condition with respect to $\mathrm{DF}_{4}$.

$\mathrm{SC7}$ : When the velocities in each phase are equal, the model $\mathrm{TF}_{4}$ satisfies the subcharacteristic condition with respect to $\mathrm{TF}_{5}$.

Herein, the results SC5 and SC7 are new to this paper and follow directly from (5.29) and (5.31). The remaining results follow from $[15,29]$. Note that it is the limit of equal phasic velocities that is of interest here. Due to the expected loss of hyperbolicity for unequal phase velocities, there is little reason to expect that the conditions SC4 and SC7 will hold more generally. This is also nontrivial to check, due to the high algebraic complexity of the eigenvalues when the velocities are not equal.

This observation is another indication that a satisfactory mathematical framework for this kind of two-phase models is still lacking. As a consequence of this, ad hoc regularization terms are commonly introduced into the models. In the next section, we discuss the consequence of introducing one such term in the present model.

\section{Condition FOR HYPERBolicity}

The canonical model derived above, with $\Delta p=0$, is generally not hyperbolic. Identically to the two-fluid six-equation model, the eigenvalues related to the volume-fraction waves are complex as soon as the gas and liquid velocities are different from each other $[19,45]$. The pressure difference term $\Delta p$ has been added to make the model hyperbolic. In order to find an expression for $\Delta p$, we will use a perturbation method around the state where $v_{\mathrm{g}}=v_{\ell}$. Based on the experience from the two-fluid six-equation model $[8,13,32,35,45]$, we look for it in the form $\Delta p=\mathcal{C} \cdot\left(v_{\mathrm{g}}-v_{\ell}\right)^{2}$. This formulation also generalizes to multifluid models [24].

We know, from the section above, the speed of sound of the model, $c_{\mathrm{TF} 4}$. The variable defined as

$$
\varepsilon=\frac{v_{\mathrm{g}}-v_{\ell}}{2 \cdot c_{\mathrm{TF}} 4}
$$

is small for subsonic velocities and is therefore suitable as a perturbation parameter. We first evaluate the characteristic polynomial

$$
\Pi_{A}=\operatorname{Det}\left(\boldsymbol{A}(\boldsymbol{U})-\lambda \cdot \boldsymbol{I}_{4}\right),
$$

where $\boldsymbol{I}_{4}$ is the identity matrix of rank 4 . In this polynomial, we make a variable change through

$$
\lambda=\frac{v_{\mathrm{g}}+v_{\ell}}{2}+a \cdot c_{\mathrm{TF} 4},
$$


where $a$ is the new unknown. Then, all the occurrences of the velocity are eliminated by substituting

$$
\begin{aligned}
& v_{\mathrm{g}}=v_{\mathrm{m}}+\varepsilon \cdot c_{\mathrm{TF} 4}, \\
& v_{\ell}=v_{\mathrm{m}}-\varepsilon \cdot c_{\mathrm{TF} 4},
\end{aligned}
$$

where $v_{\mathrm{m}}$ is the arithmetic average of $v_{\mathrm{g}}$ and $v_{\ell}$. This is in compliance with the definition of $\varepsilon(6.1)$.

Now, we perform a power-series expansion of the eigenvalues in terms of the degree of $\varepsilon$. To do so, the variable $a$ is substituted by

$$
a=\sum_{i=0}^{N}\left(b_{i} \cdot \varepsilon^{i}\right)
$$

where $N$ must be higher than the highest degree of $\varepsilon$ that we wish in the expansion. Then we will sequentially solve

$$
\text { degree }\left(\Pi_{A}, \varepsilon, i\right)=0
$$

for the coefficients $b_{i}$, starting from $i=0$, where degree $\left(\Pi_{A}, \varepsilon, i\right)$ returns the coefficient of the $i$ th degree of $\varepsilon$ in $\Pi_{A}(\varepsilon)$.

The zeroth degree gives a fourth order equation in $b_{0}$,

$$
\frac{\rho_{\mathrm{g}}^{4} \rho_{\ell}^{4}\left(\alpha_{\ell} \rho_{\mathrm{g}}+\alpha_{\mathrm{g}} \rho_{\ell}\right)^{4} L^{4}}{\left(\rho_{\mathrm{g}}-\rho_{\ell}\right)^{8} c_{\mathrm{TF} 4}^{4}}\left(b_{0}-1\right)\left(b_{0}+1\right) b_{0}^{2}=0,
$$

whose four solutions are $b_{0}=-1, b_{0}=1$, and twice $b_{0}=0$. The first two give the approximate eigenvalues

$$
\lambda=\frac{v_{\mathrm{g}}+v_{\ell}}{2} \pm c_{\mathrm{TF} 4}+\mathcal{O}\left(\frac{v_{\mathrm{g}}-v_{\ell}}{2 \cdot c_{\mathrm{TF} 4}}\right)
$$

which are clearly the eigenvalues related to the pressure waves. The double solution $b_{0}=0$ corresponds to the volume-fraction waves, which are of interest here. For this wave family, we push to the next degree of the expansion. However, the first degree of the polynomial $\Pi_{A}(\varepsilon)$ vanishes when $b_{0}=0$. We then go to the second degree. Fortunately, $b_{2}$ vanishes from the second degree, and we are left with a second order equation in $b_{1}$

$$
\left(\left(\alpha_{\mathrm{g}} \rho_{\ell}+\alpha_{\ell} \rho_{\mathrm{g}}\right) b_{1}^{2}+2\left(\alpha_{\mathrm{g}} \rho_{\ell}-\alpha_{\ell} \rho_{\mathrm{g}}\right) b_{1}+\left(\alpha_{\mathrm{g}} \rho_{\ell}+\alpha_{\ell} \rho_{\mathrm{g}}\right)-4 \mathcal{C}\right) \cdot \frac{\rho_{\mathrm{g}}^{4} \rho_{\ell}^{4} L^{4}\left(\alpha_{\mathrm{g}} \rho_{\ell}+\alpha_{\ell} \rho_{\mathrm{g}}\right)^{3}}{c_{\mathrm{TF} 4}^{4}\left(\rho_{\mathrm{g}}-\rho_{\ell}\right)^{8}}=0 .
$$

The reduced discriminant of the equation is

$$
\begin{aligned}
\Delta & =\left(\alpha_{\mathrm{g}} \rho_{\ell}-\alpha_{\ell} \rho_{\mathrm{g}}\right)^{2}-\left(\alpha_{\mathrm{g}} \rho_{\ell}+\alpha_{\ell} \rho_{\mathrm{g}}\right)\left(\alpha_{\mathrm{g}} \rho_{\ell}+\alpha_{\ell} \rho_{\mathrm{g}}-4 \mathcal{C}\right) \\
& =-4 \alpha_{\mathrm{g}} \alpha_{\ell} \rho_{\mathrm{g}} \rho_{\ell}+4\left(\alpha_{\mathrm{g}} \rho_{\ell}+\alpha_{\ell} \rho_{\mathrm{g}}\right) \mathcal{C} .
\end{aligned}
$$

Therefore $b_{1}$ will only be real if

$$
\mathcal{C} \geq \frac{\alpha_{\mathrm{g}} \alpha_{\ell} \rho_{\mathrm{g}} \rho_{\ell}}{\alpha_{\mathrm{g}} \rho_{\ell}+\alpha_{\ell} \rho_{\mathrm{g}}}
$$

which is the same constraint as the one obtained for the six-equation model [45]. The solutions are then

$$
b_{1}=\frac{-\alpha_{\mathrm{g}} \rho_{\ell}+\alpha_{\ell} \rho_{\mathrm{g}} \pm 2 \sqrt{-\alpha_{\mathrm{g}} \alpha_{\ell} \rho_{\mathrm{g}} \rho_{\ell}+\left(\alpha_{\mathrm{g}} \rho_{\ell}+\alpha_{\ell} \rho_{\mathrm{g}}\right) \mathcal{C}}}{\alpha_{\mathrm{g}} \rho_{\ell}+\alpha_{\ell} \rho_{\mathrm{g}}} .
$$

This gives the approximate eigenvalues for the volume-fraction waves

$$
\lambda=\frac{v_{\mathrm{g}}+v_{\ell}}{2}+\frac{-\alpha_{\mathrm{g}} \rho_{\ell}+\alpha_{\ell} \rho_{\mathrm{g}} \pm 2 \sqrt{-\alpha_{\mathrm{g}} \alpha_{\ell} \rho_{\mathrm{g}} \rho_{\ell}+\left(\alpha_{\mathrm{g}} \rho_{\ell}+\alpha_{\ell} \rho_{\mathrm{g}}\right) \mathcal{C}}}{\alpha_{\mathrm{g}} \rho_{\ell}+\alpha_{\ell} \rho_{\mathrm{g}}} \frac{v_{\mathrm{g}}}{2}+\mathcal{O}\left(\frac{v_{\mathrm{g}}-v_{\ell}}{2 \cdot c_{\mathrm{TF} 4}}\right) .
$$


We deduce from the above that the model with the regularising term expressed as

$$
\Delta p=\frac{\alpha_{\mathrm{g}} \alpha_{\ell} \rho_{\mathrm{g}} \rho_{\ell}}{\alpha_{\mathrm{g}} \rho_{\ell}+\alpha_{\ell} \rho_{\mathrm{g}}}\left(v_{\mathrm{g}}-v_{\ell}\right)^{2}
$$

is hyperbolic at first order around the state where $v_{\mathrm{g}}=v_{\ell}$. The same expression was previously derived for other models $[8,13,32,35,45]$. To make them actually hyperbolic when $v_{\mathrm{g}} \neq v_{\ell}$, the pressure difference in these models has commonly been defined as

$$
\Delta p=\delta \frac{\alpha_{\mathrm{g}} \alpha_{\ell} \rho_{\mathrm{g}} \rho_{\ell}}{\alpha_{\mathrm{g}} \rho_{\ell}+\alpha_{\ell} \rho_{\mathrm{g}}}\left(v_{\mathrm{g}}-v_{\ell}\right)^{2}
$$

where $\delta>1[8,13,32,35]$.

This analysis shows that the most common approach used to regularize similar models is equally valid here.

\section{Conclusion}

We have investigated a two-fluid four-equation model arising as the limit of a five-equation model when the phase relaxation becomes instantaneous. The phase relaxation source terms involve an interfacial momentum velocity, for which we found an expression respecting the second law of thermodynamics. This model was then put in quasilinear form by deriving the differentials of the primary variables. By this, we have extended previous works $[40,44]$ where these terms were treated as instantaneous relaxation source terms. This formulation has some advantages, including the possibility of using some standard numerical schemes for hyperbolic systems, following for instance the approach of [30].

We have placed our model in a hierarchy of two-phase flow relaxation models, and extracted its intrinsic speed of sound. Extending on previous works, we have shown that for smooth solutions a subcharacteristic condition holds for each relaxation process in this hierarchy. A major result of our paper is the simple and general relation (5.27) between the sound velocities of the various relaxation models. In our paper, this relation is obtained from a direct calculation and it is far from obvious why it should hold.

Acknowledgements. Both authors gratefully acknowledge partial financial support from the SINTEF group. The work of the first author has been produced with support from the BIGCCS Centre, performed under the Norwegian research program Centres for Environment-friendly Energy Research (FME). This author acknowledges the following partners for their contributions: Aker Solutions, ConocoPhillips, Det Norske Veritas, Gassco, Hydro, Shell, Statoil, TOTAL, GDF SUEZ and the Research Council of Norway (193816/S60). The second author was supported in part by the Research Council of Norway (234126/E30) through the SIMCOFLOW project. We are thankful to our colleagues Peder Aursand, Morten Hammer, Gaute Linga, Svend Tollak Munkejord and Marica Pelanti for useful discussions. We are also grateful to the anonymous reviewers who suggested significant improvements to the first version of this paper.

\section{REFERENCES}

[1] G. Allaire, S. Clerc and S. Kokh, A five-equation model for the simulation of interfaces between compressible fluids. J. Comput. Phys. 181 (2002) 577-616.

[2] G. Allaire, G. Faccanoni and S. Kokh, A strictly hyperbolic equilibrium phase transition model. C. R. Acad. Sci. Paris Ser. I 344 (2007) 135-140.

[3] M.R. Baer and J.W. Nunziato, A two-phase mixture theory for the deflagration-to-detonation transition (DDT) in reactive granular materials. Int. J. Multiphase Flow 12 (1986) 861-889.

[4] K.H. Bendiksen, D. Malnes, R. Moe and S. Nuland, The dynamic two-fluid model OLGA: Theory and application. SPE Prod. Eng. 6 (1991) 171-180.

[5] M. Bernard, S. Dellacherie, G. Faccanoni, B. Grec and Y. Penel, Study of a low Mach nuclear core model for two-phase flows with phase transition I: stiffened gas law. ESAIM: M2AN 48 (2014) 1639-1679.

[6] D. Bestion, The physical closure laws in the CATHARE code. Nucl. Eng. Des. 124 (1990) 229-245. 
[7] F. Bouchut, A reduced stability condition for nonlinear relaxation to conservation laws. J. Hyperbol. Differ. Eq. 1 (2004) $149-170$.

[8] C.-H. Chang and M.-S. Liou, A robust and accurate approach to computing compressible multiphase flow: Stratified flow model and AUSM $^{+}$-up scheme. J. Comput. Phys. 225 (2007) 850-873.

[9] G.-Q. Chen, C.D. Levermore and T.-P. Liu, Hyperbolic conservation laws with stiff relaxation terms and entropy. Commun. Pure Appl. Math. 47 (1994) 787-830.

[10] F. Coquel, K. El Amine and E. Godlewski, A numerical method using upwind schemes for the resolution of two-phase flows. J. Comput. Phys. 136 (1997) 272-288.

[11] F. Coquel, T. Gallouët, J.M. Hérard and N. Seguin, Closure laws for a two-fluid two-pressure model. C. R. Acad. Sci. Paris Ser. I 334 (2002) 927-932.

[12] J. Cortes, A. Debussche and I. Toumi, A density perturbation method to study the eigenstructure of two-phase flow equation systems. J. Comput. Phys. 147 (1998) 463-484.

[13] S. Evje and T. Flåtten, Hybrid Flux-Splitting Schemes for a Common Two-Fluid Model. J. Comput. Phys. 192 (2003) 175-210.

[14] G. Faccanoni, S. Kokh and G. Allaire, Modelling and simulation of liquid-vapor phase transition in compressible flows based on thermodynamical equilibrium. ESAIM: M2AN 46 (2012) 1029-1054.

[15] T. Flåtten and H. Lund, Relaxation two-phase flow models and the subcharacteristic condition. Math. Models Methods Appl. Sci. 21 (2011) 2379-2407.

[16] T. Flåtten and A. Morin, On Interface Transfer Terms in Two-Fluid Models. Int. J. Multiphase Flow 45 (2012) $24-29$.

[17] T. Flåtten, A. Morin and S.T. Munkejord, Wave propagation in multicomponent flow models. SIAM J. Appl. Math. 70 (2010) $2861-2882$.

[18] T. Gallouët, J.-M. Hérard and N. Seguin, Numerical modeling of two-phase flows using the two-fluid two-pressure approach. Math. Models Methods Appl. Sci. 14 (2004) 663-700.

[19] D. Gidaspow, Modeling of two-phase flow. Round table discussion (RT-1-2). Proc. of 5th Int. Heat Transfer Conf. VII (1974) 163.

[20] M. Hammer and A. Morin, A method for simulating two-phase pipe flow with real equations of state. Comput. Fluids 100 (2014) 45-58.

[21] P. Helluy and H. Mathis, Pressure laws and fast Legendre transform. Math. Models Methods Appl. Sci. 21 (2011) 745-775.

[22] P. Helluy and N. Seguin, Relaxation models of phase transition flows. ESAIM: M2AN 40 (2006) 331-352.

[23] K.H. Karlsen, C. Klingenberg and N.H. Risebro, A relaxation system for conservation laws with a discontinuous coefficient. Math. Comput. 73 (2004) 1235-1259.

[24] A. Kumbaro and M. Ndjinga, Influence of interfacial pressure term on the hyperbolicity of a general multifluid model. $J$. Comput. Multiphase Flows 3 (2011) 177-195.

[25] G. Linga, A hierarchy of non-equilibrium two-phase flow models. Submitted (2015).

[26] T.-P. Liu, Hyperbolic conservation laws with relaxation. Commun. Math. Phys. 108 (1987) 153-175.

[27] H. Lund, A hierarchy of relaxation models for two-phase flow. SIAM J. Appl. Math. 72 (2012) 1713-1741.

[28] H. Lund, Relaxation models for two-phase with applications to CO2 transport. Ph.D. thesis, Norwegian University of Science and Technology (2013).

[29] P.J. Martínez Ferrer, T. Flåtten and S.T. Munkejord, On the effect of temperature and velocity relaxation in two-phase flow models. ESAIM: M2AN 46 (2011) 411-442.

[30] A. Morin, T. Flåtten and S.T. Munkejord, A Roe scheme for a compressible six-equation two-fluid model. Int. J. Numer. Meth. Fluids 72 (2012) 478-504.

[31] S.T. Munkejord and M. Hammer, Depressurization of CO2-rich mixtures in pipes: Two-phase flow modelling and comparison with experiments. Int. J. Greenh. Gas Control 37 (2015) 398-411.

[32] S.T. Munkejord, S. Evje and T. Flåtten, A Musta scheme for a nonconservative two-fluid model. SIAM J. Sci. Comput. 31 (2009) 2587-2622.

[33] A. Murrone and H. Guillard, A five equation reduced model for compressible two phase flow problems. J. Comput. Phys. 202 (2005) 664-698.

[34] R. Natalini, Recent results on hyperbolic relaxation problems. Analysis of systems of conservation laws. Monogr. Surv. Pure Appl. Math. Chapman \& Hall/CRC (1999) 128-198.

[35] H. Paillère, C. Corre and J.R. García Cascales, On the extension of the $\mathrm{AUSM}^{+}$scheme to compressible two-fluid models. Comput. Fluids 32 (2003) 891-916.

[36] L. Pareschi and G. Russo, Implicit-explicit Runge-Kutta schemes and applications to hyperbolic systems with relaxation. $J$. Sci. Comput. 25 (2005) 129-155.

[37] M. Pelanti and K.-M. Shyue, A mixture-energy-consistent six-equation two-phase numerical model for fluids with interfaces, cavitation and evaporation waves. J. Comput. Phys. 259 (2014) 331-357.

[38] R. Saurel and R. Abgrall, A multiphase Godunov method for compressible multifluid and multiphase flows. J. Comput. Phys. 150 (1999) 425-467.

[39] R. Saurel, F. Petitpas and R. Abgrall, Modelling phase transition in metastable liquids: application to cavitating and flashing flows. J. Fluid Mech. 607 (2008) 313-350.

[40] A.L. Schor, M.S. Kazimi and N.E. Todreas, Advances in two-phase flow modeling for LMFBR applications. Nucl. Eng. Des. $82(1984)$ 127-155. 
[41] U. Setzmann and W. Wagner, A new equation of state and tables of thermodynamic properties for methane covering the range from the melting line to $625 \mathrm{~K}$ at pressures up to $1000 \mathrm{MPa}$. J. Phys. Chem. Ref. Data 20 (1991) 1061-1151.

[42] S. Solem, P. Aursand and T. Flåtten, Wave dynamics of linear hyperbolic relaxation systems. J. Hyperbol. Differ. Eq. 12 (2015) 655-670.

[43] R. Span and W. Wagner, A New Equation of State for Carbon Dioxide Covering the Fluid Region from the Triple-Point Temperature to $1100 \mathrm{~K}$ at Pressures up to $800 \mathrm{MPa}$. J. Phys. Chem. Ref. Data 25 (1996) 1509-1596.

[44] H.B. Stewart and B. Wendroff, Two-phase flow: Models and methods. J. Comput. Phys. 56 (1984) 363-409.

[45] J.H. Stuhmiller, The influence of interfacial pressure forces on the character of two-phase flow model equations. Int. J. Multiphase Flow 3 (1977) 551-560.

[46] I. Toumi, An Upwind Numerical Method for Two-Fluid Two-Phase Flow Models. Nucl. Sci. Eng. 123 (1996) $147-168$.

[47] I. Toumi and A. Kumbaro, An Approximate Linearized Riemann Solver for a Two-Fluid Model. J. Comput. Phys. 124 (1996) $286-300$.

[48] Q.H. Tran, M. Baudin and F. Coquel, A relaxation method via the Born-Infeld system. Math. Models Methods Appl. Sci. 19 (2009) 1203-1240.

[49] WАHАЗ Code Manual. JSI Report IJS-DP-8841, Jožef Stefan Insitute, Ljubljana, Slovenia (2004). 